\title{
Convexity package for momentum maps on contact manifolds
}

\author{
RIVER CHIANG \\ YAEL KARSHON
}

\begin{abstract}
Let a torus $T$ act effectively on a compact connected cooriented contact manifold, and let $\Psi$ be the natural momentum map on the symplectization. We prove that, if $\operatorname{dim} T>2$, the union of the origin with the image of $\Psi$ is a convex polyhedral cone, the nonzero level sets of $\Psi$ are connected (while the zero level set can be disconnected), and the momentum map is open as a map to its image. This answers a question posed by Eugene Lerman, who proved similar results when the zero level set is empty. We also analyze examples with $\operatorname{dim} T \leq 2$.
\end{abstract}

53D10, 53D20; 52B99

\section{Introduction}

One of the fundamental theorems in equivariant symplectic geometry is the convexity theorem for Hamiltonian torus actions. This theorem is part of the following "convexity package". Let $\Phi: M \rightarrow \mathbb{R}^{k}$ be a momentum map for a Hamiltonian torus action on a compact connected symplectic manifold. Then $\Phi$ has these properties:

(S1) The image $\Phi(M)$ is a convex polytope.

(S2) The level sets of $\Phi$ are connected.

(S3) The map $\Phi$ is open as a map to its image.

See Atiyah [2], Guillemin and Sternberg [17] and Sjamaar [34].

Eugene Lerman [24] gave an analogous theorem in equivariant contact geometry when the torus orbits are transverse to the contact distribution and asked whether the transversality condition is necessary (cf Remark 1.2). In this paper we answer Lerman's question and give a "convexity package" for momentum maps on contact manifolds. More precisely, let $M$ be a compact connected cooriented contact manifold, equipped with an effective action of a torus of dimension $k>2$, and let

$$
\Psi: M \times \mathbb{R}_{>0} \rightarrow \mathbb{R}^{k}
$$


be the momentum map on the symplectization (see below). The momentum cone is

$$
C(\Psi):=\{0\} \cup \Psi\left(M \times \mathbb{R}_{>0}\right) .
$$

Then $\Psi$ has these properties:

(C1) The momentum cone $C(\Psi)$ is a convex polyhedral cone.

(C2) The nonzero level sets, $\Psi^{-1}(\mu)$, for $\mu \neq 0$, are connected.

(C3) The map $\Psi$ is open as a map to its image.

See Theorem 9.12.

We now recall relevant definitions.

Let $M$ be a manifold of dimension $2 n+1$. A contact form on $M$ is a one-form $\alpha$ such that $\alpha \wedge(d \alpha)^{n}$ never vanishes, or, equivalently, such that $d \alpha$ is nondegenerate on $\operatorname{ker} \alpha$. If $\alpha$ is a contact one-form and $f$ is a positive function, then $f \alpha$ is a contact one-form and $\operatorname{ker} \alpha=\operatorname{ker} f \alpha$.

A contact structure $\xi$ on $M$ is a codimension one distribution (subbundle of the tangent bundle $T M$ ) that can be locally obtained as the kernel of a contact one-form. If $\xi$ is cooriented, there exists a globally defined one-form $\alpha$ such that $\xi=\operatorname{ker} \alpha$ and $\alpha$ induces the coorientation of $\xi$. Such $\alpha$ is unique up to multiplication by a positive function.

The symplectization of $(M, \alpha)$ is the symplectic manifold $\left(M \times \mathbb{R}_{>0}, d(t \alpha)\right)$, where $t$ is the coordinate on $\mathbb{R}_{>0}$ and where we use the same symbol $\alpha$ to denote the contact one-form on $M$ and its pullback to $M \times \mathbb{R}_{>0}$. Nondegeneracy of $d(t \alpha)$ follows from the property $\alpha \wedge(d \alpha)^{n} \neq 0$.

Consider the positive connected component of the annihilator of $\xi$ in the cotangent bundle $T^{*} M$ :

$\xi_{+}^{0}:=\left\{(x, \beta) \mid x \in M, \beta \in T_{x}^{*} M, \beta\left(\left.\xi\right|_{x}\right)=0, \beta\right.$ induces the coorientation of $\left.\left.\xi\right|_{x}\right\}$.

The map $M \times \mathbb{R}_{>0} \rightarrow \xi_{+}^{0}$ which sends $(x, t)$ to $t \alpha_{x}$ defines a trivialization of $\xi_{+}^{0}$ as a principal $\mathbb{R}_{>0}$ bundle. Also, it pulls back the tautological one-form on $T^{*} M$ to the one-form $t \alpha$ on $M \times \mathbb{R}_{>0}$ and the standard symplectic form on $T^{*} M$ to the symplectic form $d(t \alpha)$ on $M \times \mathbb{R}_{>0}$. Thus, to avoid choosing a contact one-form, one may define the symplectization of $(M, \xi)$ to be the symplectic submanifold $\xi_{+}^{0}$ of $T^{*} M$.

Let a torus $T \cong\left(S^{1}\right)^{k}$ act on $M$ and preserve the cooriented distribution $\xi$. Let $X_{M}$, for $X$ in the Lie algebra $\mathfrak{t}$ of $T$, denote the vector fields on $M$ that are induced from the 
action: $X_{M}(x)=\left.\frac{d}{d t}\right|_{t=0}(\exp t X) \cdot x$. The action naturally lifts to a Hamiltonian action on the cotangent bundle $T^{*} M$ which preserves the submanifold $\xi_{+}^{0}$. The standard momentum map on $T^{*} M$ restricts to a momentum map on $\xi_{+}^{0}$ whose $X$ component, for $X \in \mathfrak{t}$, is given by $(x, \beta) \mapsto \beta\left(X_{M}(x)\right)$ for all $x \in M$ and $\left.\beta \in \xi_{+}^{0}\right|_{x} \subset T_{x}^{*} M$.

Let $\alpha$ be a $T$-invariant contact one-form on $M$. (Such $\alpha$ can be obtained by averaging; see Lemma 2.6 of Lerman's paper [25].) The $\alpha$-momentum map is the map $\Psi_{\alpha}: M \rightarrow \mathfrak{t}^{*}$ whose $X$ component $\Psi_{\alpha}^{X}: M \rightarrow \mathbb{R}$, for $X \in \mathfrak{t}$, is given by

$$
\Psi_{\alpha}^{X}(x)=\alpha\left(X_{M}(x)\right)
$$

for all $x \in M$. It satisfies $d \Psi_{\alpha}^{X}=-\iota\left(X_{M}\right) d \alpha$ on $M$. Using $\alpha$ to identify $\xi_{+}^{0}$ with $M \times \mathbb{R}_{>0}$, the induced $T$ action on $M \times \mathbb{R}_{>0}$ is the given action on the $M$ component and is trivial on the $\mathbb{R}_{>0}$ component, and the momentum map becomes the map

$$
\Psi: M \times \mathbb{R}_{>0} \rightarrow t^{*}, \quad(x, t) \mapsto t \Psi_{\alpha}(x),
$$

which we call the contact momentum map corresponding to $\alpha$. Thus, the momentum cone is

$$
\begin{aligned}
C(\Psi) & =\{0\} \cup \Psi\left(M \times \mathbb{R}_{>0}\right) \\
& =\mathbb{R}_{\geq 0} \cdot \Psi_{\alpha}(M) .
\end{aligned}
$$

1.2 Remark The action is said to be transverse if the orbits are transverse to the contact distribution. By the formula (1.1) for the momentum map, an action is transverse if and only if its momentum map never takes the value zero. A contact momentum map $\Psi: M \times \mathbb{R}_{>0} \rightarrow t^{*}$ is never proper as a map to $t^{*}:$ the preimage of a closed ball centered at the origin is never compact. But if $M$ is compact and the action is transverse, then the momentum map is proper as a map to $t^{*} \backslash\{0\}$. In the case of transverse torus actions, parts (3) and (4) of Theorem 9.12 were proved by Lerman in [24].

1.3 Remark The definition of the contact momentum map, as a map on $M \times \mathbb{R}_{>0}$, depends on the choice of one-form $\alpha$. The topological properties of the contact momentum map are independent of the choice of $\alpha$, because we can work directly on $\xi_{+}^{0}$.

1.4 Remark If the image of the contact momentum map is contained in an open half-space, then the "convexity package" $(\mathrm{C} 1),(\mathrm{C} 2),(\mathrm{C} 3)$ is true without the dimension assumption on $T$, and it follows from the "convexity package" for symplectic manifolds with proper momentum maps: 
Let $\Phi: Q \rightarrow \mathfrak{t}^{*}$ be a momentum map for a torus action on a connected symplectic manifold. Suppose that there exists a convex subset $\mathcal{T}$ of $\mathfrak{t}^{*}$ that contains the image $\Phi(Q)$ and such that $\Phi: Q \rightarrow \mathcal{T}$ is proper. Then $\Phi$ is open as a map to its image, the image $\Phi(Q)$ is a convex polyhedral subset of $\mathcal{T}$, and the level sets of $\Phi$ are connected.

See Condevaux, Dazord and Molino [12], Hilgert, Neeb and Plank [19], Prato [32], Lerman, Meinrenken, Tolman and Woodward [26, Theorem 4.3] and Bjorndahl and Karshon [9, Theorem 30].

1.5 Remark Under our assumptions that guarantee (C1), (C2) and (C3) (in particular, $\operatorname{dim} T>2$ ), the zero level set can be disconnected; see Examples 10.3 and 10.4. Lerman noted, in [24, Remark 1.4] and referring to his construction in [23], that if $\operatorname{dim} T \leq 2$, then (C2) may fail, and if $\operatorname{dim} T=2$, then (C1) may fail. We analyze Lerman's examples in more detail in Example 10.6, where $\operatorname{dim} T=2$ and the zero level set is empty, and in Example 10.7, where $\operatorname{dim} T=1$ and the zero level set is nonempty. If $\operatorname{dim} T=1$ and the zero level set is empty, (C1), (C2), (C3) all hold, by Remark 1.4. In fact, (C1) holds trivially if $\operatorname{dim} T=1$. If $\operatorname{dim} T=2$ and the zero level set is nonempty, we are currently unaware of examples where (C1), (C2) and (C3) don't all hold.

The "convexity package" (C1), (C2), (C3) says that the momentum map has certain global topological properties. To prove it, we show that the momentum map has certain local topological properties, and we show that these local properties imply the required global properties by means of a point-set-topological "local to global" argument. We now give some details.

Let $X$ denote a compact connected Hausdorff space.

We define a map $\varphi: X \rightarrow \mathbb{R}^{n}$ to be convex if any two points in $X$ can be connected by a path whose composition with $\varphi$ is a weakly monotone parametrization of a (possibly degenerate) segment. See Section 2. Similarly, we define a map $\Psi: X \rightarrow S^{n-1}$ to be spherically convex if any two points in $X$ whose images are not antipodal can be connected by a path whose composition with $\Psi$ is a weakly monotone geodesic of length $<\pi$. The image of such a map is spherically convex, and the level sets of such a map are connected. See Remark 3.1 and Definition 4.1.

In Sections 3-5 we give a "local to global" argument for maps from $X$ to $S^{n-1}$. If the image is not contained in a great circle, and if every point has a neighborhood on which the map is spherically convex and open to its image, then the map is spherically convex and open to its image. This result, together with the analysis of the case that the image is contained in a great circle, is given in Proposition 5.3. 
In Section 6, we give a "local to global" argument for a map $\Psi: X \times \mathbb{R}_{>0} \rightarrow \mathbb{R}^{n}$ that is obtained from a nonvanishing map $\varphi: X \rightarrow \mathbb{R}^{n}$ by $\Psi(x, \lambda)=\lambda \varphi(x)$. Suppose that the image is not contained in a two dimensional subspace, and suppose that every point in $X \times \mathbb{R}_{>0}$ has a neighborhood on which $\Psi$ is convex and $\Psi /\|\Psi\|$ is open to its image. Then every two points $y_{0}$ and $y_{1}$ in $X \times \mathbb{R}_{>0}$ for which the segment $\left[\Psi\left(y_{0}\right), \Psi\left(y_{1}\right)\right]$ does not contain the origin can be connected by a path whose composition with $\Psi$ is a weakly monotone parametrization of this segment. Additionally, $\Psi$ is open as a map to its image. See Proposition 6.8.

In Section 7 we define $\Psi$ as before but we allow $\varphi$ to sometimes vanish. This is the case that applies to contact momentum maps for nontransverse actions. The strategy is to remove from $X$ the $\varphi$-preimage of an open ball around the origin and to apply the results of Section 6 to the resulting "excised space". The precise statement is more technical than in the nonvanishing case, and the proof is more involved. We formulate local assumptions on $\varphi$ and $\Psi$ that guarantee the following global properties. Every two points in $X \times \mathbb{R}_{>0}$ that do not both lie on the zero level set can be connected by a path whose composition with $\Psi$ is a weakly monotone parametrization of a (possibly degenerate) segment; additionally, the map $\Psi$ is open as a map to its image. See Proposition 7.11.

In Section 9 we show that momentum maps on symplectizations have the local openness and convexity properties that, by the results of Section 7, imply the global properties that we had set to prove. These local properties are consequences of the local normal form theorem, which describes the neighborhood of an orbit in a symplectic manifold with a Hamiltonian action of a compact Lie group. We apply the local normal form theorem in two different ways:

(1) The symplectization is a symplectic manifold with a Hamiltonian torus action. We apply the local normal form theorem to neighborhoods of its orbits.

(2) Take the contact manifold itself, with a momentum map associated to some choice of invariant contact one-form. Consider an orbit that lies on the zero level set of the momentum map. By formula (1.1), the tangent space to this orbit is contained in the contact distribution; therefore it is transverse to the Reeb directions. We find an invariant codimension one submanifold containing the orbit and transverse to the Reeb directions, on which the differential of the contact form is a nondegenerate two-form. We apply the local normal form to this submanifold.

To show the local properties of the momentum map, it remains to examine the local models that appear in the local normal form theorem. We use the additional fact that 
the momentum map image of the orbit belongs to the annihilator of the stabilizer of the orbit; it holds because the momentum map comes from a one-form whose differential is the symplectic form.

By examining the local models, we need to show that the restriction of a linear projection to a standard simplex with a facet removed is open as a map to its image and has the weak path lifting property. These properties may be intuitively obvious but their rigorous proofs are not entirely trivial; we give them in Section 8.

We conclude Section 9 with a proof of the convexity package; see Theorem 9.12. And, in Section 10, we conclude the paper with examples.

The type of local-to-global technique that we use was initiated by Condevaux, Dazord and Molino [12] and developed by Hilgert, Neeb and Plank [19;20], Birtea, Ortega and Ratiu [8; 7], and Bjorndahl and Karshon [9]; also see Knop [22]. It can be viewed as a generalization of the Tietze-Nakajima theorem [30; 35].

Convexity results in contact geometry appeared in Banyaga and Molino [3] (toric case) and in Lerman [24] (transverse case). Torus actions on contact manifolds (or on symplectic cones, cf Remark 10.1) were also studied by Guillemin and Sternberg [18], Albert [1], Kamishima and Tsuboi [21], de Moraes and Tomei [29], Boyer and Galicki [10], Loose [28], Lerman [23; 24; 25], Lerman and Willett [27], Willett [36] and Nozawa [31].

The book [16] by Guillemin and Sjamaar gives an overview of convexity results in symplectic geometry. The book [15] by Geiges is a general reference for (not necessarily equivariant) contact geometry. Finally, our notion of convexity is unrelated to "contact convexity" as in, eg, [15, Chapter 5].

Acknowledgements We are grateful to Eugene Lerman for teaching us about momentum maps for contact manifolds. YK is grateful to Shlomo Sternberg for directing her to the paper by Condevaux, Dazord and Molino, many years ago.

This research was partially supported by an NSERC Discovery grant, by the NSC grant 96-2115-M-006-010-MY2 and by the NCTS (South).

\section{Convex maps}

2.1 Definition A path $\gamma:[a, b] \rightarrow \mathbb{R}^{n}$ is weakly monotone straight if, for any $t_{1}, t_{2}, t_{3} \in[a, b]$, if $t_{1} \leq t_{2} \leq t_{3}$, then $\gamma\left(t_{2}\right) \in\left[\gamma\left(t_{1}\right), \gamma\left(t_{3}\right)\right]$. 
2.2 Definition A map $\varphi$ from a topological space $X$ to $\mathbb{R}^{n}$ or to a convex subset of $\mathbb{R}^{n}$ is a convex map if every two points $x_{0}, x_{1}$ in $X$ can be connected by a path $\gamma:[0,1] \rightarrow X$ with $\gamma(0)=x_{0}$ and $\gamma(1)=x_{1}$, such that the composition $\varphi \circ \gamma:[0,1] \rightarrow$ $\mathbb{R}^{n}$ is weakly monotone straight.

Clearly, if $\varphi: X \rightarrow \mathbb{R}^{n}$ is convex, then $X$ is connected, the image of $\varphi$ is convex, and the level sets of $\varphi$ are connected.

Remark Our definition of a convex map, from a topological space to $\mathbb{R}^{n}$, does not agree with the notion of a "convex function", from a convex subset of a vector space to $\mathbb{R}$, being a function that satisfies $f(t x+(1-t) y) \leq t f(x)+(1-t) f(y)$ for all $0 \leq t \leq 1$.

We recall Theorem 15 of [9]:

2.3 Theorem Let $X$ be a connected Hausdorff topological space, let $\mathcal{T}$ be a convex subset of $\mathbb{R}^{n}$, and let $\Psi: X \rightarrow \mathcal{T}$ be a continuous proper map. Suppose that for every point $x \in X$ there exists an open neighborhood $U$ of $x$ such that the map $\left.\Psi\right|_{U}: U \rightarrow \Psi(U)$ is convex and open. Then the map $\Psi: X \rightarrow \Psi(X)$ is convex and open.

2.4 Example In each of the following examples, $X$ is a subset of $\mathbb{R}^{2}$, and $\Psi: X \rightarrow \mathbb{R}$ is the projection to the $x$-coordinate. In each of these examples, exactly one of the assumptions of Theorem 2.3 fails, and the map $\Psi$ is not convex.

(1) Let $X$ be the union of the set where $x>0$ and $|y| \leq 1$ and the set where $x=0$ and $|y|=1$. The projection to the $x$-axis is locally convex and locally open to its image, but it is not proper.

(2) Let $X$ be the union of the negative $x$-axis, the set where $x \geq 0$ and $y=x$, and the set where $x \geq 0$ and $y=-x$. The projection to the $x$-axis is locally open and is proper, but it is not locally convex.

(3) Let $X$ be the union of the negative $x$-axis, the portion of the $y$-axis where $|y|<1$, and the set where $x \geq 0$ and $|y|=1$. The projection to the $x$-axis is locally convex and is proper, but it is not locally open to its image.

\section{Spherical geometry}

In this section we recall some elementary facts from spherical geometry. 
Let $S^{n-1}$ be the unit sphere in $\mathbb{R}^{n}$. A great circle in $S^{n-1}$ is an intersection of $S^{n-1}$ with a two dimensional plane through the origin in $\mathbb{R}^{n}$. A path $\gamma:[0,1] \rightarrow S^{n-1}$ is a weakly monotone geodesic if it is either constant or is equal to a composition

$$
[0,1] \longrightarrow\left[\theta_{0}, \theta_{1}\right] \stackrel{(\cos (\cdot), \sin (\cdot))}{\longrightarrow} S^{1} \stackrel{\iota}{\longrightarrow} S^{n-1}
$$

where the map $[0,1] \rightarrow\left[\theta_{0}, \theta_{1}\right] \subset \mathbb{R}$ is onto and weakly monotone and where the map $\iota$ is an isometric embedding of $S^{1}$ into $S^{n-1}$ as a great circle. The length of the path is equal to the length of the interval $\left[\theta_{0}, \theta_{1}\right]$. The path is a weakly monotone short geodesic if its length is $<\pi$. A subset of $S^{n-1}$ is spherically convex if every short geodesic whose endpoints belong to the set is contained in the set. An open hemisphere is the intersection of $S^{n-1}$ with an open half-space whose boundary contains the origin. An open hemisphere is spherically convex. More generally, let $w$ be a point of $S^{n-1}$ and let $B(w, \varepsilon)$ denote the open ball of radius $\varepsilon$ centered at $w$. If $B(w, \varepsilon) \cap S^{n-1}$ is contained in an open hemisphere, then it is spherically convex.

3.1 Remark There are several inequivalent notions of convexity in the literature; see, eg, Danzer, Grünbaum and Klee [13, Section 9.1]. "Robinson convexity" [33] is defined for closed subsets of the sphere, and, for these subsets, it coincides with our notion of spherical convexity. Note that this notion allows a set that consists of exactly one pair of antipodal points. The definitions of "geodesically convex" are stricter; the entire sphere is spherically convex according to our definition but it is not geodesically convex.

3.2 Lemma Let $\gamma:[0,1] \rightarrow \mathbb{R}^{n}$ be a weakly monotone straight path that does not pass through the origin. Then $\gamma_{S}:=\gamma /\|\gamma\|:[0,1] \rightarrow S^{n-1}$ is a weakly monotone short geodesic.

The geometric intuition behind the lemma should be clear. We give an algebraic proof.

Proof If $\gamma(\cdot)$ is contained in a line through the origin, $\gamma_{S}(\cdot)$ is constant.

Suppose that $\gamma(\cdot)$ is not contained in a line through the origin. By choosing appropriate coordinates on the plane that contains the set $\{0\} \cup\{\gamma(t)\}$, we may assume that $n=2$ and that there exists $c>0$ such that $\gamma(\cdot)$ is contained in the horizontal line $\{(\cdot, c)\}$. Because $\gamma$ is weakly monotone straight, there exist real numbers $a, b$ and a weakly monotone function $s:[0,1] \rightarrow[0,1]$ such that $s(0)=0, s(1)=1$, and $\gamma(t)=((1-s(t)) a+s(t) b, c)$. Then $\gamma_{S}(t)=(\cos \theta(t), \sin \theta(t))$ where

$$
\theta(t)=\operatorname{Arctan} \frac{c}{(1-s(t)) a+s(t) b} \in(0, \pi)
$$

Because $t \mapsto s(t)$ is weakly monotone, so is the denominator of (3.3), as is $t \mapsto \theta(t)$. 
We now give a converse result:

3.4 Lemma Let $\gamma_{S}:[0,1] \rightarrow S^{n-1}$ be a weakly monotone short geodesic. Let $\lambda_{0}$ and $\lambda_{1}$ be positive numbers. Then there exists a weakly monotone straight path $\gamma:[0,1] \rightarrow \mathbb{R}^{n}$ such that $\gamma(0)=\lambda_{0} \gamma_{S}(0), \gamma(1)=\lambda_{1} \gamma_{S}(1)$, and $\gamma(t) /\|\gamma(t)\|=\gamma_{S}(t)$.

Proof If $\gamma_{S}(\cdot)$ is constant, set $\gamma(t)=(1-t) \lambda_{0} \gamma_{S}(0)+t \lambda_{1} \gamma_{S}(1)$.

Suppose that $\gamma_{S}(\cdot)$ is not constant. By choosing appropriate coordinates on the plane that contains the set $\{0\} \cup\left\{\gamma_{S}(t)\right\}$, we may assume that $n=2$ and that there exists a positive number $c$ such that $\lambda_{0} \gamma_{S}(0)$ and $\lambda_{1} \gamma_{S}(1)$ both belong to the horizontal line $\{(\cdot, c)\}$.

Because $\gamma_{S}(\cdot)$ is a weakly monotone geodesic that is contained in the upper half plane, there exists a weakly monotone function $\theta:[0,1] \rightarrow(0, \pi)$ such that $\gamma_{S}(t)=$ $(\cos \theta(t), \sin \theta(t))$. Project along radii to the line $\{(\cdot, c)\}$ : set

$$
\gamma(t)=(c \cot \theta(t), c) .
$$

Then $t \mapsto \gamma(t)$ is weakly monotone straight, and $\gamma /\|\gamma\|=\gamma_{S}$. Also, $\gamma(0)=\lambda_{0} \gamma_{S}(0)$ and $\gamma(1)=\lambda_{1} \gamma_{S}(1)$.

As before, $B(w, \varepsilon)$ denotes the open ball of radius $\varepsilon$ centered at $w$.

We recall an elementary fact that relates straightness on spheres to straightness in vector spaces:

3.5 Lemma There exists a homeomorphism $A$, from the upper hemisphere

$$
S_{+}^{n-1}=\left\{\left(x_{1}, x_{2}, \ldots, x_{n}\right) \in S^{n-1} \mid x_{n}>0\right\}
$$

onto $\mathbb{R}^{n-1}$, with the following properties. Denote the north pole by $w_{0}$.

(1) For any $\varepsilon>0$, the map $A$ carries the "cap" $B\left(w_{0}, \varepsilon\right) \cap S_{+}^{n-1}$ onto a ball centered at the origin in $\mathbb{R}^{n-1}$.

(2) Let $\gamma$ be a path in $S_{+}^{n-1}$. Then $\gamma$ is a weakly monotone geodesic if and only if $A \circ \gamma$ is a straight line segment in $\mathbb{R}^{n-1}$ with a weakly monotone parametrization.

Proof We set $A$ to be the projection from $S_{+}^{n-1}$ to the hyperplane $\{(\cdot, \ldots, \cdot, 1)\}$ along rays emanating from the origin, followed by the projection $\mathbb{R}^{n-1} \times\{1\} \rightarrow \mathbb{R}^{n-1}$. That is, $A\left(x_{1}, \ldots, x_{n-1}, x_{n}\right)=\left(x_{1} / x_{n}, \ldots, x_{n-1} / x_{n}\right)$. This is a homeomorphism with inverse

$$
\left(y_{1}, \ldots, y_{n-1}\right) \mapsto \frac{1}{\sqrt{y_{1}^{2}+\ldots+y_{n-1}^{2}+1}}\left(y_{1}, \ldots, y_{n-1}, 1\right) .
$$


It maps the north pole $\omega_{0}$ to the origin, and a cap $B\left(w_{0}, \varepsilon\right) \cap S_{+}^{n-1}$ to a ball centered at the origin in $\mathbb{R}^{n-1}$. If $P$ is a 2-plane through the origin in $\mathbb{R}^{n}$, the map $A$ carries the great half-circle $P \cap S_{+}^{n-1}$ to the straight line $P \cap\left\{x_{n}=1\right\}$.

Every weakly monotone geodesic in $S_{+}^{n-1}$ has the form

$$
t \mapsto(\alpha \cos \theta(t), \sin \theta(t))
$$

where $\alpha$ is in $S^{n-2}$ and $\theta:[0,1] \rightarrow(0, \pi)$ is weakly monotone. The map $A$ carries this geodesic to the path

$$
t \mapsto \alpha \cot \theta(t) .
$$

Because the function $\cot (\cdot)$ is weakly monotone, the path is weakly monotone straight. Thus, the map $A$ carries a weakly monotone short geodesic in $S_{+}^{n-1}$ to a weakly monotone straight path in $\mathbb{R}^{n-1}$. Conversely, the inverse map, $A^{-1}(x)=(x, 1) /\|(x, 1)\|$, carries a weakly monotone straight path in $\mathbb{R}^{n-1} \cong \mathbb{R}^{n-1} \times\{1\}$ to a weakly monotone short geodesic in $S_{+}^{n-1}$, by Lemma 3.2.

We now recall a property of spherical triangles:

3.6 Lemma In a spherical right triangle contained in a half-sphere $S_{+}^{n-1}$, if one leg has length $>\pi / 2$, then that leg is longer than the hypotenuse.

Proof Given a spherical triangle in $S_{+}^{n-1}$, its vertices span a three dimensional linear subspace; the intersection of this subspace with $S_{+}^{n-1}$ is a two dimensional hemisphere that contains the triangle. Hence it suffices to prove the lemma for a spherical triangle in $S_{+}^{2}$.

Let $A, B, C$ denote the vertices of a spherical triangle in $S_{+}^{2}$ and $a, b, c$ their facing arc lengths. Then $0<a, b, c<\pi$. Suppose that the angle at $C$ is a right angle and that $a>\pi / 2$. The fundamental formula of spherical trigonometry [6, Section 18.6] implies that $\cos c=\cos a \cos b$, which implies that $c \leq \pi / 2<a$ if $b \geq \pi / 2$, and $\pi / 2<c<a$ if $b<\pi / 2$. Therefore, the leg $a>\pi / 2$ is always longer than the hypotenuse $c$.

\section{Spherically convex maps}

We now consider maps to spheres. In this section, for simplicity, we restrict attention to the sphere of radius one. In later sections we take the freedom to use the same terminology and results for spheres of arbitrary radii.

Recall that $B(w, \varepsilon)$ denotes the open ball of radius $\varepsilon$ centered at $w$. Let $B_{\varepsilon}$ be a shorthand for $B(0, \varepsilon)$ and $\partial B_{\varepsilon}$ be its boundary. 
4.1 Definition A map $\psi$ from a topological space $X$ to the sphere $S^{n-1}$ is a spherically convex map if for every two points $x_{0}$ and $x_{1}$ in $X$, if $\psi\left(x_{1}\right) \neq-\psi\left(x_{0}\right)$, then there exists a path $\gamma:[0,1] \rightarrow X$ such that $\gamma(0)=x_{0}, \gamma(1)=x_{1}$, and the composition $\psi \circ \gamma:[0,1] \rightarrow S^{n-1}$ is a weakly monotone short geodesic.

4.2 Remark A subset $X$ of $S^{n-1}$ is spherically convex in the sense of Section 3 exactly if the inclusion map $\psi: X \rightarrow S^{n-1}$ is a spherically convex map.

4.3 Remark Suppose that $\psi: X \rightarrow S^{n-1}$ is spherically convex and let $A$ be a subset of $\mathbb{R}^{n}$. If $A \cap S^{n-1}$ is spherically convex, then the map $\left.\psi\right|_{\psi^{-1}(A)}$ is spherically convex. If, additionally, $A \cap S^{n-1}$ does not consist of a single pair of antipodal points, then $\psi^{-1}(A)$ is connected. In particular, if $H$ is an open half-space whose boundary contains the origin, then $\left.\psi\right|_{\psi^{-1}(H)}$ is spherically convex and $\psi^{-1}(H)$ is connected. More generally, if $w \in S^{n-1}$ and if $\varepsilon>0$ is sufficiently small so that $B(w, \varepsilon) \cap S^{n-1}$ is contained in an open hemisphere, then $\left.\psi\right|_{\psi^{-1}(B(w, \varepsilon))}$ is spherically convex and $\psi^{-1}(B(w, \varepsilon))$ is connected.

We will adjust arguments of [9] to our needs. To start, we have the following variant of Proposition 17 of [9].

4.4 Lemma Let $\varphi$ be a continuous map from a Hausdorff topological space to $\mathbb{R}^{n}$. Let $K$ be a compact connected subset of the level set $\varphi^{-1}(0)$. Suppose that each point $x$ in $K$ has an open neighborhood $U_{x}$ such that the map $\left.\varphi\right|_{U_{x}}: U_{x} \rightarrow \varphi\left(U_{x}\right)$ is convex and open. Then there exists an open neighborhood $U_{K}$ of $K$ such that the map $\left.\varphi\right|_{U_{K}}: U_{K} \rightarrow \varphi\left(U_{K}\right)$ is convex and open.

Moreover, we can choose $U_{K}$ such that the following conditions hold.

- Suppose that for each $x$ in $K$ and each sufficiently small $\delta>0$, every point in $\varphi^{-1}\left(\partial B_{\delta}\right) \cap U_{x}$ has a neighborhood $V \subset U_{x}$ such that the restriction $\left.\varphi\right|_{\varphi^{-1}\left(\partial B_{\delta}\right) \cap V}$ is spherically convex. Then we can choose $U_{K}$ so that, for sufficiently small $\delta>0$, every point in $\varphi^{-1}\left(\partial B_{\delta}\right) \cap U_{K}$ has a neighborhood $U \subset U_{K}$ such that $\left.\varphi\right|_{\varphi^{-1}\left(\partial B_{\delta}\right) \cap U}$ is also spherically convex.

- Suppose that for each $x$ in $K$ there exists a cone $C_{x}$ with vertex at the origin such that $\varphi\left(U_{x}\right)$ is an open subset of $C_{x}$. Then the cones $C_{x}$ are all equal to each other, and we can choose $U_{K}$ so that $\varphi\left(U_{K}\right)$ is also an open subset of this common cone.

4.5 Remark A cone with vertex at the origin is a set that is invariant under multiplication by positive numbers; cf [5, Section 11.1.6]. Some authors require a cone to also contain its vertex; $\mathrm{cf}[4, \mathrm{II} .(8.1)]$. We only use this term for sets that already contain the origin. 
Proof of Lemma 4.4 Let $U_{1}, \ldots, U_{N}$ be open sets such that

$$
\begin{gathered}
U_{i} \cap K \neq \varnothing, \\
K \subset U_{1} \cup \cdots \cup U_{N}
\end{gathered}
$$

and $\left.\varphi\right|_{U_{i}}: U_{i} \rightarrow \varphi\left(U_{i}\right)$ is convex and open.

Suppose $U_{i} \cap U_{j} \cap K \neq \varnothing$. Then $\varphi\left(U_{i} \cap U_{j}\right)$ contains 0 . Since $U_{i} \cap U_{j}$ is open in $U_{i}$ and the map $\left.\varphi\right|_{U_{i}}: U_{i} \rightarrow \varphi\left(U_{i}\right)$ is open, there exists a positive number $\varepsilon_{i j}$ such that $\varphi\left(U_{i} \cap U_{j}\right) \cap B_{\varepsilon_{i j}}=\varphi\left(U_{i}\right) \cap B_{\varepsilon_{i j}}$.

Let $\varepsilon=\min \left\{\varepsilon_{i j} \mid U_{i} \cap U_{j} \cap K \neq \varnothing\right\}$. Then $\varphi\left(U_{i}\right) \cap B_{\varepsilon}=\varphi\left(U_{j}\right) \cap B_{\varepsilon}=\varphi\left(U_{i} \cap U_{j}\right) \cap B_{\varepsilon}$ whenever $U_{i} \cap U_{j} \cap K \neq \varnothing$.

Because $K$ is connected, every $U_{i}$ and $U_{j}$ can be connected by a sequence $U_{i}=$ $U_{i_{0}}, U_{i_{1}}, \ldots, U_{i_{s}}=U_{j}$ such that $U_{i_{\ell-1}} \cap U_{i_{\ell}} \cap K \neq \varnothing$ for $\ell=1, \ldots, s$. So the set $\varphi\left(U_{i}\right) \cap B_{\varepsilon}$ is the same for all $i$ and is equal to $\varphi\left(U_{i} \cap U_{j}\right) \cap B_{\varepsilon}$ whenever $U_{i} \cap U_{j} \cap K \neq \varnothing$. Call this set $W$. Let

$$
U_{K}=\left(U_{1} \cup \cdots \cup U_{N}\right) \cap \varphi^{-1}\left(B_{\varepsilon}\right) .
$$

Then

$$
\begin{aligned}
\varphi\left(U_{K}\right) & =\varphi\left(U_{i}\right) \cap B_{\varepsilon} & & \text { for all } i \\
& =\varphi\left(U_{i} \cap U_{j}\right) \cap B_{\varepsilon} & & \text { if } U_{i} \cap U_{j} \cap K \neq \varnothing \\
& =W . & &
\end{aligned}
$$

The level sets of $\left.\varphi\right|_{U_{K}}: U_{K} \rightarrow W$ are connected. This follows from the following facts:

(1) For every $U_{i}$, every level set of $\left.\varphi\right|_{U_{K}}: U_{K} \rightarrow W$ meets $U_{i}$.

(2) Whenever $U_{i} \cap U_{j} \cap K \neq \varnothing$, every level set of $\left.\varphi\right|_{U_{K}}: U_{K} \rightarrow W$ meets $U_{i} \cap U_{j}$.

(3) Every $U_{i}$ and $U_{j}$ can be connected by a sequence $U_{i}=U_{i_{0}}, U_{i_{1}}, \ldots, U_{i_{s}}=U_{j}$ such that $U_{i_{\ell-1}} \cap U_{i_{\ell}} \cap K \neq \varnothing$.

(4) The level sets of each $\left.\varphi\right|_{U_{i}}$ are connected.

We now show that $\left.\varphi\right|_{U_{K}}$ is convex. Let $x_{0}$ and $x_{1}$ be points in $U_{K}$. Then $x_{0}$ is contained in some $U_{i}$. Because $\varphi\left(U_{i}\right)$ contains $\varphi\left(U_{K}\right)$, there exists a point $\bar{x}_{1}$ in $U_{i}$ with $\varphi\left(\bar{x}_{1}\right)=\varphi\left(x_{1}\right)$; because $\left.\varphi\right|_{U_{i}}$ is convex, there exists a path in $U_{i}$ from $x_{0}$ to $\bar{x}_{1}$ whose composition with $\varphi$ is weakly monotone straight and whose image in $\mathbb{R}^{n}$ is $\left[\varphi\left(x_{0}\right), \varphi\left(x_{1}\right)\right]$. Because the level sets of $\left.\varphi\right|_{U_{K}}$ are connected, there exists a path from $\bar{x}_{1}$ to $x_{1}$ that is contained in $\varphi^{-1}\left(\varphi\left(x_{1}\right)\right)$; the concatenation of the path from $x_{0}$ to $\bar{x}_{1}$ with the path from $\bar{x}_{1}$ to $x_{1}$ is a path from $x_{0}$ to $x_{1}$ whose composition with $\varphi$ is weakly monotone straight with image $\left[\varphi\left(x_{0}\right), \varphi\left(x_{1}\right)\right]$. This shows that $\left.\varphi\right|_{U_{K}}$ is convex. 
Since the map $\left.\varphi\right|_{U_{i} \cap \varphi^{-1}\left(B_{\varepsilon}\right)}: U_{i} \cap \varphi^{-1}\left(B_{\varepsilon}\right) \rightarrow W$ is open for every $i$, the map $\left.\varphi\right|_{U_{K}}: U_{K} \rightarrow W$ is open.

Suppose that $\delta_{i}>0$ is such that, for all $0<\delta<\delta_{i}$, every point in $\varphi^{-1}\left(\partial B_{\delta}\right) \cap U_{i}$ has a neighborhood $V \subset U_{i}$ such that the restriction $\left.\varphi\right|_{\varphi^{-1}\left(\partial B_{\delta}\right) \cap V}$ is spherically convex. Then, for every $0<\delta<\min \left\{\varepsilon, \delta_{1}, \ldots, \delta_{N}\right\}$, because $U_{K} \cap \varphi^{-1}\left(\partial B_{\delta}\right)$ is the union of the sets $U_{i} \cap \varphi^{-1}\left(\partial B_{\delta}\right)$, every point in $U_{K} \cap \varphi^{-1}\left(\partial B_{\delta}\right)$ has a neighborhood $U$ (namely, $U=V \cap \varphi^{-1}\left(B_{\varepsilon}\right)$ for some $\left.V \subset U_{i}\right)$ such that the restriction of $\varphi$ to $U \cap \varphi^{-1}\left(\partial B_{\delta}\right)$ is spherically convex.

Suppose that $\varphi\left(U_{i}\right)$ is an open subset of a cone with vertex at the origin. Then, because $\varphi\left(U_{i}\right) \cap B_{\varepsilon}=W$, this cone is equal to $\mathbb{R}_{>0} \cdot W$, and $W$ is open in the cone. In particular, the cone is independent of $i$. Then $\varphi\left(U_{K}\right)$, being also equal to $W$, is an open subset of this cone.

4.6 Notation Fix a map $\psi: X \rightarrow S^{n-1}$. For $x \in X$ with $\psi(x)=w$, we denote by $[x]$ the connected component of $x$ in $\psi^{-1}(w)$, and we denote by $U_{[x], \varepsilon}$ the connected component of $x$ in $\psi^{-1}(B(w, \varepsilon))$.

We now give another variant of Proposition 17 of [9], which applies to a spherical map:

4.7 Lemma Let $X$ be a compact Hausdorff topological space, and let $\psi: X \rightarrow S^{n-1}$ be a continuous map. Suppose that every point in $X$ is contained in an open set $U$ such that the map $\left.\psi\right|_{U}: U \rightarrow \Phi(U)$ is open and is spherically convex. Then for every

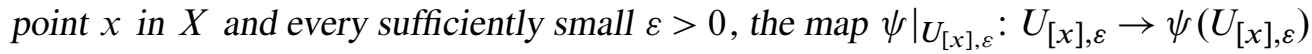
is open and spherically convex.

Proof Fix $x \in X$. Without loss of generality, assume that $w:=\psi(x)$ is the north pole. Let $S_{+}^{n-1}$ denote the open upper hemisphere, let $A: S_{+}^{n-1} \rightarrow \mathbb{R}^{n-1}$ be the homeomorphism of Lemma 3.5, and let $X_{+}=\psi^{-1}\left(S_{+}^{n-1}\right)$. Using Lemma 3.5, and applying Lemma 4.4 to the composition $\left.A \circ \psi\right|_{X_{+}}: X_{+} \rightarrow \mathbb{R}^{n-1}$ and the set $K=[x]$, we find a neighborhood $V$ of $[x]$ that is contained in $X_{+}$and such that the map $\psi: V \rightarrow \psi(V)$ is open and spherically convex.

We now show that there exists $\varepsilon>0$ such that the neighborhood $V$ of $[x]$ contains the connected component $U_{[x], \varepsilon}$ of $[x]$ in $\psi^{-1}(B(w, \varepsilon))$.

Because $\psi$ is proper, the level set $\psi^{-1}(w)$ is compact. Because every point in $X$ has a neighborhood on which $\psi$ (is spherically convex, hence) has connected level sets, the level set $\psi^{-1}(w)$ is locally connected. These two properties imply that the level set $\psi^{-1}(w)$ has only finitely many connected components. Because these components are 
compact and disjoint and $X$ is Hausdorff, there exist disjoint open subsets $\mathcal{O}_{1}, \ldots, \mathcal{O}_{k}$ of $X$ such that every $\mathcal{O}_{j}$ contains exactly one component of $\psi^{-1}(w)$. Without loss of generality, suppose that the component $[x]$ is contained in the set $\mathcal{O}_{1}$. Let $\mathcal{O}_{1}^{\prime}=\mathcal{O}_{1} \cap V$. Because $\psi$ is proper and $\mathcal{O}_{1}^{\prime} \cup \mathcal{O}_{2} \cup \cdots \mathcal{O}_{k}$ is a neighborhood of the level set $\psi^{-1}(w)$, there exists $\varepsilon>0$ such that $\psi^{-1}(B(w, \varepsilon))$ is contained in $\mathcal{O}_{1}^{\prime} \cup \mathcal{O}_{2} \cup \cdots \mathcal{O}_{k}$. Because the sets $\mathcal{O}_{1}^{\prime}, \mathcal{O}_{2}, \ldots, \mathcal{O}_{k}$ are open and disjoint and the set $U_{[x], \varepsilon}$ is connected and meets $\left([x]\right.$, hence) $\mathcal{O}_{1}^{\prime}$, the set $U_{[x], \varepsilon}$ is entirely contained in $\mathcal{O}_{1}^{\prime}$. In particular, $U_{[x], \varepsilon}$ is contained in $V$. Choosing $\varepsilon$ sufficiently small, we may assume that $B(w, \varepsilon) \cap S^{n-1}$ is contained in an open hemisphere.

Because $\left.\psi\right|_{V}$ is spherically convex, by Remark 4.3, $V \cap \psi^{-1}(B(w, \varepsilon))$ is connected. Since $V \cap \psi^{-1}(B(w, \varepsilon))$ is a connected subset of $\psi^{-1}(B(w, \varepsilon))$ that contains the connected component $U_{[x], \varepsilon}$, it must be equal to this component: $V \cap \psi^{-1}(B(w, \varepsilon))=$ $U_{[x], \varepsilon}$. By this and Remark 4.3, since $\left.\psi\right|_{V}$ is spherically convex and open to its image, so is $\left.\psi\right|_{U_{[x], \varepsilon}}$.

4.8 Remark A similar result holds with more general target spaces; one can avoid Lemma 3.5 and work with an analogue of Lemma 4.4 for more general target spaces. See Birtea, Ortega and Ratiu [7]. We will not need this level of generality.

We let $l(\gamma) \in[0, \infty]$ denote the length of a continuous curve $\gamma:[0,1] \rightarrow \mathbb{R}^{n}$.

We now have the following variant of Theorem 15 of [9].

4.9 Proposition Let $X$ be a compact connected Hausdorff topological space and let

$$
\psi: X \rightarrow S^{n-1}
$$

be a continuous map. Suppose that every point of $X$ is contained in an open set $U \subset X$ such that the map $\left.\psi\right|_{U}: U \rightarrow \psi(U)$ is open and is spherically convex. Then every two points in $X$ can be connected by a path $\gamma$ such that $\psi \circ \gamma$ is a weakly monotone geodesic and such that $l(\psi \circ \gamma) \leq l\left(\psi \circ \gamma^{\prime}\right)$ for every other path $\gamma^{\prime}$ connecting the two points.

The following proof of Proposition 4.9 is analogous to those of Theorem 15 and Lemma 23 of [9], which follow Condevaux, Dazord and Molino [12].

Proof of Proposition 4.9 For two points $x$ and $x^{\prime}$ in $X$, define $d_{\psi}\left(x, x^{\prime}\right)$ to be the infimum of the lengths $l(\psi \circ \gamma)$ as $\gamma$ varies over all paths in $X$ from $x$ to $x^{\prime}$.

Note that if $x^{\prime} \in U_{[x], \varepsilon / 2}$ and $d_{\psi}\left(x^{\prime}, x^{\prime \prime}\right) \leq \varepsilon / 2$, then $x^{\prime \prime} \in U_{[x], \varepsilon}$ (cf Notation 4.6). 
Let $x_{0}$ and $x_{1}$ be points of $X$. We show that there exists a "midpoint" $x_{1 / 2}$ such that

$$
d_{\psi}\left(x_{0}, x_{1 / 2}\right)=d_{\psi}\left(x_{1 / 2}, x_{1}\right)=\frac{1}{2} d_{\psi}\left(x_{0}, x_{1}\right) .
$$

Choose paths $\gamma_{n}$ connecting $x_{0}$ to $x_{1}$ such that the sequence of lengths $l\left(\psi \circ \gamma_{k}\right)$ converges to $d_{\psi}\left(x_{0}, x_{1}\right)$. Let $t_{k} \in[0,1]$ be such that $l\left(\left.\psi \circ \gamma_{k}\right|_{\left[0, t_{k}\right]}\right)=l\left(\left.\psi \circ \gamma_{k}\right|_{\left[t_{k}, 1\right]}\right)=$ $\frac{1}{2} l\left(\psi \circ \gamma_{k}\right)$. Since $X$ is compact, there exists a point $x_{1 / 2}$ such that every neighborhood of $x_{1 / 2}$ contains $\gamma_{k}\left(t_{k}\right)$ for infinitely many values of $k$. Let $\varepsilon>0$. There exists $j$ such that $\gamma_{j}\left(t_{j}\right)$ belongs to a neighborhood $U$ of $x_{1 / 2}$ on which $\psi$ is spherically convex, such that $d_{\psi}\left(\gamma_{j}\left(t_{j}\right), x_{1 / 2}\right)<\varepsilon / 2$, and such that $l\left(\psi \circ \gamma_{j}\right)<d_{\psi}\left(x_{0}, x_{1}\right)+\varepsilon$. The path $\left.\gamma_{j}\right|_{\left[0, t_{j}\right]}$, followed by a path in $U$ from $\gamma_{j}\left(t_{j}\right)$ to $x_{1 / 2}$ whose composition with $\psi$ is a weakly monotone geodesic, form a path from $x_{0}$ to $x_{1 / 2}$ whose composition with $\psi$ has length $<\frac{1}{2} d_{\psi}\left(x_{0}, x_{1}\right)+\varepsilon$. This implies that $d_{\psi}\left(x_{0}, x_{1 / 2}\right) \leq \frac{1}{2} d_{\psi}\left(x_{0}, x_{1}\right)$. Similarly, $d_{\psi}\left(x_{1 / 2}, x_{1}\right) \leq \frac{1}{2} d_{\psi}\left(x_{0}, x_{1}\right)$.

Iterating, we find points $x_{j / 2^{m}}$, for $0 \leq j \leq 2^{m}$, such that

$$
d_{\psi}\left(x_{j_{1} / 2^{m}}, x_{j_{2} / 2^{m}}\right)=\frac{\left|j_{1}-j_{2}\right|}{2^{m}} \quad \text { for all } j_{1}, j_{2} \in\left\{0,1, \ldots, 2^{m}\right\} .
$$

By Lemma 4.7, and since $X$ is compact, there exist points $\bar{x}_{1}, \ldots, \bar{x}_{N}$ and positive numbers $\varepsilon_{1}, \ldots, \varepsilon_{N}$ such that $\left.\psi\right|_{U_{\left[\bar{x}_{i}\right], \varepsilon_{i}}}$ is open to its image and is spherically convex and such that the sets $U_{\left[\bar{x}_{i}\right], \varepsilon_{i} / 2}$ cover $X$. Let $\varepsilon=\min \left\{\varepsilon_{1}, \ldots, \varepsilon_{N}\right\}$. Then for every $x^{\prime}$ and $x^{\prime \prime}$, if $d_{\psi}\left(x^{\prime}, x^{\prime \prime}\right) \leq \varepsilon / 2$, then there exists a path from $x^{\prime}$ to $x^{\prime \prime}$ whose composition with $\psi$ is a weakly monotone short geodesic.

Choose $m$ large enough so that $\left(1 / 2^{m}\right) d_{\psi}\left(x_{0}, x_{1}\right)<\frac{1}{4} \varepsilon$. Then each pair among the three points $x_{(j-1) / 2^{m}}, x_{j / 2^{m}}, x_{(j+1) / 2^{m}}$ can be connected by a path whose composition with $\psi$ is a weakly monotone short geodesic. Because

$$
d_{\psi}\left(x_{(j-1) / 2^{m}}, x_{j / 2^{m}}\right)+d_{\psi}\left(x_{j / 2^{m}}, x_{(j+1) / 2^{m}}\right)=d_{\psi}\left(x_{(j-1) / 2^{m}}, x_{(j+1) / 2^{m}}\right),
$$

these paths fit into a path from $x_{0}$ to $x_{1}$ whose composition with $\psi$ is a weakly monotone geodesic. Because the length of this geodesic is $d_{\psi}\left(x_{0}, x_{1}\right)$, it is shorter than $\psi \circ \gamma^{\prime}$ for any other path $\gamma^{\prime}$ connecting $x_{0}$ to $x_{1}$.

We have this easy consequence of Proposition 4.9:

4.11 Corollary Under the assumptions of Proposition 4.9, let $x_{0}$ and $x_{1}$ be points of $X$, and suppose that there exists a path $\tilde{\gamma}$ in $X$ from $x_{0}$ to $x_{1}$ such that $l(\psi \circ \tilde{\gamma})<\pi$. Then there exists a path $\gamma$ in $X$ from $x_{0}$ to $x_{1}$ such that $\psi \circ \gamma$ is a short geodesic. 
4.12 Remark Let $\psi: X \rightarrow S^{n-1}$ be as in Proposition 4.9. As in Condevaux-DazordMolino [12], consider the equivalence relation on $X$ in which $x_{0} \sim x_{1}$ if and only if there exists a path $\gamma:[0,1] \rightarrow X$ such that $\gamma(0)=x_{0}$ and $\gamma(1)=x_{1}$ and such that $\psi \circ \gamma$ is constant. The function $d_{\psi}$ defined in the proof of Proposition 4.9 descends to a metric on $X / \sim$; this follows from Lemma 4.7. With this metric, $X / \sim$ is a length space. (This means that the distance between two points is equal to the infimum of the lengths of curves that connect them. As usual, the length of a continuous curve $\{\gamma(t)\}_{t \in[0,1]}$ is the supremum of $\sum_{i=1}^{n} \operatorname{distance}\left(\gamma\left(t_{i}\right), \gamma\left(t_{i-1}\right)\right)$ over all partitions $0=t_{0}<t_{1}<\ldots<t_{n}=1$.) Proposition 4.9 then follows from the Hopf-Rinow theorem for length spaces. See Bridson and Haefliger [11, Chapter I.3].

\section{Shortening a nonminimal monotone geodesic}

5.1 Proposition Let $X$ be a compact connected Hausdorff space. Let $\psi: X \rightarrow S^{n-1}$ be a continuous map. Suppose that each point in $X$ is contained in an open set $U \subset X$ such that $\left.\psi\right|_{U}: U \rightarrow \psi(U)$ is spherically convex and is open.

Let $\gamma:[0,1] \rightarrow X$ be a path in $X$ such that $\psi \circ \gamma$ is a weakly monotone geodesic of length $\pi<l(\psi \circ \gamma)<2 \pi$. Let $E \subset S^{n-1}$ be the great circle that contains this geodesic; suppose that the image $\psi(X)$ is not entirely contained in $E$. Then there exists a path $\tilde{\gamma}:[0,1] \rightarrow X$, with the same endpoints as $\gamma$, such that $l(\psi \circ \tilde{\gamma})<l(\psi \circ \gamma)$.

Proof Let $t_{1 / 2}$ be such that $l\left(\left.\psi \circ \gamma\right|_{\left[0, t_{1 / 2}\right]}\right)=l\left(\left.\psi \circ \gamma\right|_{\left[t_{1 / 2}, 1\right]}\right)=(1 / 2) l(\psi \circ \gamma)$. Let $x_{0}=\gamma(0), x_{1 / 2}=\gamma\left(t_{1 / 2}\right), x_{1}=\gamma(1)$. Because $\left.\psi \circ \gamma\right|_{\left[0, t_{1 / 2}\right]}$ and $\left.\psi \circ \gamma\right|_{\left[t_{1 / 2}, 1\right]}$ are short geodesics, $d_{\psi}\left(x_{0}, x_{1 / 2}\right)=d_{\psi}\left(x_{1 / 2}, x_{1}\right)=(1 / 2) l(\psi \circ \gamma)$.

By Proposition 4.9 and because the image $\Psi(X)$ is not contained in $E$, we can connect $x_{1 / 2}$ to a point outside $\psi^{-1}(E)$ by a path $\hat{\gamma}$ whose composition with $\psi$ is a weakly monotone geodesic.

For each $\varepsilon>0$ sufficiently small, let $\hat{x}_{\varepsilon}$ be a point on the path $\hat{\gamma}$ so that $d_{\psi}\left(x_{1 / 2}, \hat{x}_{\varepsilon}\right)=$ $\varepsilon$, and let $x_{t_{\varepsilon}}$ be a point on the path $\gamma$ such that $\psi\left(x_{t_{\varepsilon}}\right)$ is closest to $\psi\left(\hat{x}_{\varepsilon}\right)$ among all points on the great circle $E$. Then there is a path in $X$ from $x_{t_{\varepsilon}}$ to $\hat{x}_{\varepsilon}$ whose composition with $\psi$ is contained in a great circle that is perpendicular to $\gamma$.

We have $\lim _{\varepsilon \rightarrow 0} d_{\psi}\left(x_{t_{1 / 2}}, x_{t_{\varepsilon}}\right)=\lim _{\varepsilon \rightarrow 0} d_{\psi}\left(x_{t_{1 / 2}}, \hat{x}_{\varepsilon}\right)=0$.

Fix $\varepsilon>0$ sufficiently small so that

$$
d_{\psi}\left(x_{0}, x_{t_{\varepsilon}}\right)>\frac{\pi}{2}, \quad d_{\psi}\left(x_{1}, x_{t_{\varepsilon}}\right)>\frac{\pi}{2} \quad \text { and } \quad d_{\psi}\left(x_{1}, x_{t_{\varepsilon}}\right)+d_{\psi}\left(x_{t_{\varepsilon}}, \hat{x}_{\varepsilon}\right)<\pi .
$$


By Lemma 3.6, $d_{\psi}\left(x_{0}, \hat{x}_{\varepsilon}\right)<d_{\psi}\left(x_{0}, x_{t_{\varepsilon}}\right)$ and $d_{\psi}\left(x_{1}, \hat{x}_{\varepsilon}\right)<d_{\psi}\left(x_{1}, x_{t_{\varepsilon}}\right)$. Thus, there exists a path from $x_{0}$ to $\hat{x}_{\varepsilon}$ whose composition with $\psi$ has length $<d_{\psi}\left(x_{0}, x_{t_{\varepsilon}}\right)$, and there exists a path from $\hat{x}_{\varepsilon}$ to $x_{1}$ whose composition with $\psi$ has length $<d_{\psi}\left(x_{t_{\varepsilon}}, x_{1}\right)$. The concatenation of these paths is a path from $x_{0}$ to $x_{1}$ whose composition with $\psi$ has length $<d_{\psi}\left(x_{0}, x_{t_{\varepsilon}}\right)+d_{\psi}\left(x_{t_{\varepsilon}}, x_{1}\right)=l(\psi \circ \gamma)$.

5.2 Corollary Let $X$ be a compact connected Hausdorff topological space. Let $\psi: X \rightarrow S^{n-1}$ be a continuous map. Suppose that each point in $X$ has an open neighborhood $U$ such that $\left.\psi\right|_{U}$ is spherically convex and is open as a map to its image, $\psi(U)$. Suppose that $\psi(X)$ is not contained in a great circle. Then the map $\psi: X \rightarrow \psi(X) \subset S^{n-1}$ is spherically convex and is open as a map to its image.

Proof Let $x_{0}$ and $x_{1}$ be points of $X$ such that $\psi\left(x_{1}\right) \neq-\psi\left(x_{0}\right)$. By Proposition $4.9, x_{0}$ and $x_{1}$ can be connected by a weakly monotone geodesic that is shortest among all paths from $x_{0}$ to $x_{1}$. Since $\psi(X)$ is not contained in a great circle, by Proposition 5.1, this geodesic cannot have length $>\pi$. So it has length $\leq \pi$. Because $\psi\left(x_{1}\right) \neq-\psi\left(x_{0}\right)$, it has length $<\pi$. That is, $x_{0}$ and $x_{1}$ can be connected by a weakly monotone short geodesic. This proves that $\psi$ is spherically convex.

Fix $w \in S^{n-1}$. Since $\psi$ is spherically convex, if $\varepsilon$ is sufficiently small, $\psi^{-1}(B(w, \varepsilon))$ is connected (cf Remark 4.3). So $\psi^{-1}\left(B(w, \varepsilon)\right.$ ) is equal to $U_{[x], \varepsilon}$ for any $x \in \psi^{-1}(w)$ (cf Notation 4.6). By Lemma 4.7, if $\varepsilon$ is sufficiently small, it follows that $\left.\psi\right|_{\psi^{-1}(B(w, \varepsilon) \text { ) }}$ is open as a map to its image. Since for every $w \in S^{n-1}$ there exists $\varepsilon>0$ such that $\left.\psi\right|_{\psi^{-1}(B(w, \varepsilon))}$ is open as a map to its image, $\psi$ is open as a map to its image.

We now give the ultimate "local to global" result, for maps to spheres:

5.3 Proposition Let $X$ be a compact connected Hausdorff topological space. Let $\psi: X \rightarrow S^{n-1}$ be a continuous map. Suppose that every point in $X$ has an open neighborhood $U$ such that $\left.\psi\right|_{U}$ is spherically convex and is open as a map to its image, $\psi(U)$.

Suppose that $\psi(X)$ is not contained in a great circle. Then all the following results hold.

(1) For every $x_{0}$ and $x_{1}$ in $X$ there exists a path $\gamma$ from $x_{0}$ to $x_{1}$ such that $\psi \circ \gamma$ is a weakly monotone geodesic of length $\leq \pi$.

(2) The set $C=\mathbb{R}_{\geq 0} \cdot \psi(X)$ is a convex cone with vertex at the origin: if $w_{1}, w_{2} \in C$ and $\lambda_{1}, \lambda_{2} \in \mathbb{R}_{\geq 0}$, then $\lambda_{1} w_{1}+\lambda_{2} w_{2} \in C$.

(3) The level sets of $\psi$ are connected.

(4) The map $\psi$ is open as a map to its image. 
Suppose that the image $\psi(X)$ is contained in a great circle. Let $\iota: S^{1} \rightarrow S^{n-1}$ be an isometric parametrization of that great circle. Then exactly one of the following three possibilities occurs.

(i) There exist an interval $\left[\theta_{0}, \theta_{1}\right] \subset \mathbb{R}$, and a surjective map $\tilde{\psi}: X \rightarrow\left[\theta_{0}, \theta_{1}\right]$ which is convex and open, such that the map $\psi: X \rightarrow S^{n-1}$ is equal to the composition

$$
X \stackrel{\tilde{\psi}}{\longrightarrow}\left[\theta_{0}, \theta_{1}\right] \stackrel{(\cos (\cdot), \sin (\cdot))}{\longrightarrow} S^{1} \stackrel{\iota}{\longrightarrow} S^{n-1} .
$$

(ii) There exists a surjective map $\tilde{\psi}: X \rightarrow S^{1}$, which is spherically convex and open, and a positive integer $m$, such that the map $\psi$ is equal to the composition

$$
X \stackrel{\tilde{\psi}}{\longrightarrow} S^{1} \stackrel{(\cos \theta, \sin \theta) \mapsto(\cos m \theta, \sin m \theta)}{\longrightarrow} S^{1} \stackrel{\iota}{\longrightarrow} S^{n-1} .
$$

(iii) $\psi$ is constant.

5.4 Remark The case of Proposition 5.3 in which $\psi(X)$ is not contained in a great circle follows from Theorem 2.17 of [7] with two slight adjustments. In Definition 2.9 of [7] of local convexity data, replace "for every sufficiently small neighborhood $U_{x}$ of $x$ the set $f\left(U_{x}\right)$ is convex" by "there exist arbitrarily small neighborhoods $U_{x}$ of $x$ such that the set $f\left(U_{x}\right)$ is convex". In part (ii) of Theorem 2.17 of [7], apply the "uniquely geodesic" assumption to $f(X)$, not to $Y$.

Proof of Proposition 5.3 We first analyze the case that the image $\psi(X)$ is not contained in a great circle.

Let $x_{0}$ and $x_{1}$ be points of $X$. If $\psi\left(x_{0}\right) \neq-\psi\left(x_{1}\right)$, Part (1) follows from Corollary 5.2. If $\psi\left(x_{0}\right)=-\psi\left(x_{1}\right)$, take any $x^{\prime}$ such that $\psi\left(x^{\prime}\right)$ is different from $\psi\left(x_{0}\right)$ and $\psi\left(x_{1}\right)$. By Corollary 5.2, connect $x_{0}$ to $x^{\prime}$ and $x^{\prime}$ to $x_{1}$ by paths whose images are short geodesics. The concatenation of these paths is a path from $x_{0}$ to $x_{1}$ whose composition with $\psi$ is a weakly monotone geodesic of length $\pi$. This gives part (1).

Part (2) follows from Part (1) and Lemma 3.4.

Part (3) follows from Part (1). For any two points $x_{0}, x_{1}$ in the same level set, there exists a path $\gamma$ in $X$ connecting them such that $\psi \circ \gamma$ is a weakly monotone short geodesic. Because $\psi\left(x_{0}\right)=\psi\left(x_{1}\right)$, the composition $\psi \circ \gamma$ must be constant.

Part (4) was proved in Corollary 5.2.

We now analyze the case that the image $\psi(X)$ is contained in a great circle. Without loss of generality, assume that $n=2$ and $\iota=$ identity. By the theory of covering spaces, exactly one of the following possibilities occurs. 
(a) The image of $\pi_{1}(X)$ in $\pi_{1}\left(S^{1}\right)$ is trivial, and there exists a map $\tilde{\psi}: X \rightarrow \mathbb{R}$ such that the map $\psi: X \rightarrow S^{1}$ is equal to the composition

$$
X \stackrel{\tilde{\psi}}{\longrightarrow} \mathbb{R} \stackrel{(\cos (\cdot), \sin (\cdot))}{\longrightarrow} S^{1} .
$$

(b) The image of $\pi_{1}(X)$ in $\pi_{1}\left(S^{1}\right) \cong \mathbb{Z}$ is the subgroup of index $m$, and there exists a map $\psi: X \rightarrow S^{1}$ such that the map $\psi$ is equal to the composition

$$
X \stackrel{\tilde{\psi}}{\longrightarrow} S^{1} \stackrel{(\cos \theta, \sin \theta) \mapsto(\cos m \theta, \sin m \theta)}{\longrightarrow} S^{1}
$$

and such that the map $\tilde{\psi}_{*}: \pi_{1}(X) \rightarrow \pi_{1}\left(S^{1}\right)$ is onto.

Assume that we are in case (a).

Let $x$ be a point of $X$. Let $U$ be a neighborhood of $x$ in $X$ such that $\left.\psi\right|_{U}$ is spherically convex and is open to its image.

Let $J \subset S^{1}$ be a half-circle that contains $\psi(x)$. Then $U^{\prime}:=U \cap \psi^{-1}(J)$ is also a neighborhood of $x$ on which $\psi$ is spherically convex and is open to its image, and $U^{\prime}$ is connected.

The preimage of $J$ under the map $(\cos (\cdot), \sin (\cdot))$ is a disjoint union of segments. Because $U_{\widetilde{J}}^{\prime}$ is connected, $\widetilde{\psi}\left(U^{\prime}\right)$ is contained in one of these segments; call this segment $\widetilde{J}$.

The map $(\cos (\cdot), \sin (\cdot))$ restricts to a homeomorphism from $\widetilde{J}$ onto $J$. The map $\left.\psi\right|_{U^{\prime}}$ is the composition of $\left.\widetilde{\psi}\right|_{U^{\prime}}$ with this homeomorphism. Therefore, because $\left.\psi\right|_{U^{\prime}}$ is open to its image, so is $\left.\widetilde{\psi}\right|_{U^{\prime}}$.

A lifting to $\mathbb{R}$ of a weakly monotone geodesic in $S^{1}$ is weakly monotone. Therefore, because $\left.\psi\right|_{U^{\prime}}$ is spherically convex, $\left.\widetilde{\psi}\right|_{U^{\prime}}$ is convex.

We have shown that every point in $X$ has a neighborhood $U^{\prime}$ such that $\left.\tilde{\psi}\right|_{U^{\prime}}$ is convex and is open to its image. By Theorem 2.3 it follows that $\tilde{\psi}: X \rightarrow \mathbb{R}$ is convex and is open to its image. Because $X$ is compact, the image of $\widetilde{\psi}$ is either a single point or a closed segment. This shows that exactly one of the possibilities (i) or (iii) must occur.

Now assume that we are in case (b).

Let $x$ be a point of $X$. Let $U$ be a neighborhood of $x$ in $X$ such that $\left.\psi\right|_{U}$ is spherically convex and is open to its image.

Let $J \subset S^{1}$ be an open arc of the circle that contains $\psi(x)$ and that has length $<2 \pi / m$. Then $U^{\prime}:=U \cap \psi^{-1}(J)$ is also a neighborhood of $x$ on which $\psi$ is spherically convex and is open to its image, and $U^{\prime}$ is connected. 
The preimage of $J$ under the map $(\cos \theta, \sin \theta) \mapsto(\cos m \theta, \sin m \theta)$ is a disjoint union of $m$ arcs of $S^{1}$. Because $U^{\prime}$ is connected, $\widetilde{\psi}\left(U^{\prime}\right)$ is contained in one of these arcs; call this arc $\widetilde{J}$.

The map $(\cos (\cdot), \sin (\cdot))$ restricts to a homeomorphism from $\widetilde{J}$ onto $J$. The map $\left.\psi\right|_{U^{\prime}}$ is the composition of $\left.\widetilde{\psi}\right|_{U^{\prime}}$ with this homeomorphism. Therefore, because $\left.\psi\right|_{U^{\prime}}$ is open to its image, so is $\left.\widetilde{\psi}\right|_{U^{\prime}}$.

A lifting to $\widetilde{J}$ of a weakly monotone geodesic in $J$ is a weakly monotone geodesic in $\widetilde{J}$. Therefore, because $\left.\psi\right|_{U^{\prime}}$ is spherically convex, so is $\left.\tilde{\psi}\right|_{U^{\prime}}$.

We have shown that every point in $X$ has a neighborhood $U^{\prime}$ such that $\left.\tilde{\psi}\right|_{U^{\prime}}$ is spherically convex and is open to its image. Also, $\widetilde{\psi}$ induces a surjection $\pi_{1}(X) \rightarrow$ $\pi_{1}\left(S^{1}\right)$. It remains to show that these assumptions imply that $\tilde{\psi}: X \rightarrow S^{1}$ is spherically convex and open.

We have a commuting diagram

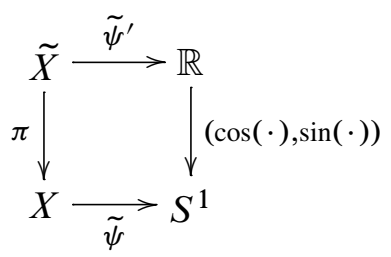

where $\tilde{X}$ is the fibered product $X \times{ }_{S^{1}} \mathbb{R}$ and $\pi: \tilde{X} \rightarrow X$ is the covering map. An argument similar to that of case (a) applied to $\widetilde{\psi} \circ \pi$ show that every point in $\tilde{X}$ has a neighborhood on which $\tilde{\psi}^{\prime}$ is open to its image and is convex. The map $\tilde{\psi}^{\prime}: \tilde{X} \rightarrow \mathbb{R}$ is proper; this follows from the fact that $X$ is compact. The space $\tilde{X}$ is connected; this follows from the assumptions that the map $\widetilde{\psi}_{*}: \pi_{1}(X) \rightarrow \pi_{1}\left(S^{1}\right)$ is onto and the map $\tilde{\psi}: X \rightarrow S^{1}$ is spherically convex. By Theorem 2.3 it follows that the map $\tilde{\psi}^{\prime}$ is open to its image and is convex. Because we are in case (b), the map $\tilde{\psi}^{\prime}$ is onto. Hence $\tilde{\psi}: X \rightarrow S^{1}$ is spherically convex and open.

\section{Local to global convexity for conification of nonvanishing functions}

6.1 Lemma Let $X$ be a Hausdorff topological space and $\varphi: X \rightarrow \mathbb{R}^{n}$ a continuous map. Define a map $\Psi: X \times \mathbb{R}_{>0} \rightarrow \mathbb{R}^{n}$ by $\Psi(x, \lambda)=\lambda \varphi(x)$. Suppose that the map $\varphi$ is convex. Then the map $\Psi$ is convex. 
Proof Let $\left(x_{0}, \lambda_{0}\right)$ and $\left(x_{1}, \lambda_{1}\right)$ be two points in $X \times \mathbb{R}_{>0}$. Because the map $\varphi$ is convex, there exists a path $x(t)$ in $X$ from $x_{0}$ to $x_{1}$ such that $\varphi(x(t))$ is weakly monotone straight. That is, there exists a weakly monotone continuous function

$$
s:[0,1] \rightarrow[0,1]
$$

such that $s(0)=0$ and $s(1)=1$ and such that

$$
\varphi(x(t))=(1-s(t)) \varphi\left(x_{0}\right)+s(t) \varphi\left(x_{1}\right) .
$$

We rewrite the right hand side as

$$
\frac{1-s(t)}{\lambda_{0}} \lambda_{0} \varphi\left(x_{0}\right)+\frac{s(t)}{\lambda_{1}} \lambda_{1} \varphi\left(x_{1}\right)
$$

and then divide both sides of the equation by the sum of the coefficients,

$$
\frac{1-s(t)}{\lambda_{0}}+\frac{s(t)}{\lambda_{1}}
$$

which is positive. Setting

$$
\begin{aligned}
\lambda(t) & =\frac{1}{\frac{1-s(t)}{\lambda_{0}}+\frac{s(t)}{\lambda_{1}}}, \\
\tilde{s}(t) & =\frac{\frac{s(t)}{\lambda_{1}}}{\frac{1-s(t)}{\lambda_{0}}+\frac{s(t)}{\lambda_{1}}},
\end{aligned}
$$

we get

$$
\lambda(t) \varphi(x(t))=(1-\widetilde{s}(t)) \lambda_{0} \varphi\left(x_{0}\right)+\widetilde{s}(t) \lambda_{1} \varphi\left(x_{1}\right) .
$$

Because $\lambda(t)$ is a path of positive numbers connecting $\lambda_{0}$ to $\lambda_{1}$, the path $y(t)=$ $(x(t), \lambda(t))$ in $X \times \mathbb{R}_{>0}$ connects $\left(x_{0}, \lambda_{0}\right)$ to $\left(x_{1}, \lambda_{1}\right)$. It remains to show that $\Psi(y(t))$ is weakly monotone straight. By (6.3), it suffices to show that $\widetilde{s}(t)$ is a weakly monotone continuous function from $[0,1]$ to $[0,1]$ such that $\widetilde{s}(0)=0$ and $\widetilde{s}(1)=1$. Because $s(\cdot)$ is a continuous function from $[0,1]$ to $[0,1]$ such that $s(0)=0$ and $s(1)=1$, and by $(6.2)$, we see that $\widetilde{s}(\cdot)$ is a continuous function from $[0,1]$ to $[0,1]$ such that $\widetilde{s}(0)=0$ and $\widetilde{s}(1)=1$. Monotonicity of $\widetilde{s}(t)$ follows from that of $s(t)$ since, whenever $s(t)>0$,

$$
\frac{1}{\widetilde{s}(t)}=1+\frac{\lambda_{1}}{\lambda_{0}}\left(\frac{1}{s(t)}-1\right) .
$$


6.4 Lemma Let $X$ be a Hausdorff topological space and $\varphi: X \rightarrow \mathbb{R}^{n}$ a continuous nonvanishing map. Define maps $\Psi: X \times \mathbb{R}_{>0} \rightarrow \mathbb{R}^{n}$ and $\bar{\Psi}: X \times \mathbb{R}_{>0} \rightarrow S^{n-1}$ by $\Psi(x, \lambda)=\lambda \varphi(x)$ and $\bar{\Psi}=\Psi /\|\Psi\|$. Also define $\psi=\varphi /\|\varphi\|: X \rightarrow S^{n-1}$.

Let $U$ be an open subset of $X \times \mathbb{R}_{>0}$. Let $V$ be the image of $U$ under the projection $X \times \mathbb{R}_{>0} \rightarrow X$.

The set $V$ is an open subset of $X$. If the map $\left.\Psi\right|_{U}$ is convex, then the map $\left.\psi\right|_{V}$ is spherically convex. If the map $\left.\bar{\Psi}\right|_{U}$ is open to its image, so is the map $\left.\psi\right|_{V}$.

Proof Openness of $V$ in $X$ follows from the definition of the product topology on $X \times \mathbb{R}_{>0}$.

The set $\bar{\Psi}(U)$ is equal to the set $\psi(V)$; call this set $W$. We have a commuting diagram of continuous maps:

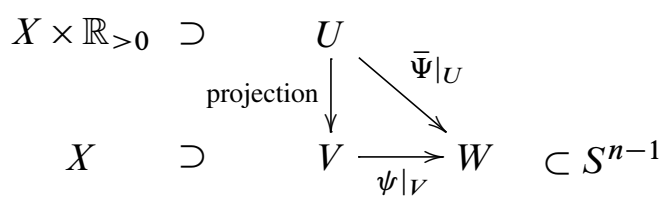

Suppose that the map $\left.\Psi\right|_{U}: U \rightarrow \mathbb{R}^{n}$ is convex. By Lemma 3.2, the map $\left.\bar{\Psi}\right|_{U}: U \rightarrow W$ is spherically convex. Because the projection map $U \rightarrow V$ is onto, and by the commuting diagram, the map $\left.\psi\right|_{V}: V \rightarrow W$ is spherically convex.

Suppose that the map $\left.\bar{\Psi}\right|_{U}: U \rightarrow W$ is open. Because the projection map $U \rightarrow V$ is onto, and by the commuting diagram, the map $\left.\psi\right|_{V}: V \rightarrow W$ is open.

6.5 Corollary Let $X$ be a Hausdorff topological space and $\varphi: X \rightarrow \mathbb{R}^{n}$ a continuous nonvanishing map. Define maps $\Psi: X \times \mathbb{R}_{>0} \rightarrow \mathbb{R}^{n}$ and $\bar{\Psi}: X \times \mathbb{R}_{>0} \rightarrow S^{n-1}$ by $\Psi(x, \lambda)=\lambda \varphi(x)$ and $\bar{\Psi}=\Psi /\|\Psi\|$. Also define $\psi=\varphi /\|\varphi\|: X \rightarrow S^{n-1}$.

Suppose that each point in $X \times \mathbb{R}_{>0}$ has a neighborhood $U$ such that the map $\left.\Psi\right|_{U}: U \rightarrow \mathbb{R}^{n}$ is convex and such that the map $\left.\bar{\Psi}\right|_{U}: U \rightarrow \bar{\Psi}(U) \subset S^{n-1}$ is open to its image.

Then each point in $X$ has a neighborhood $V$ such that the map $\left.\psi\right|_{V}$ is spherically convex and is open to its image.

6.6 Remark Let $D_{r} \subset \mathbb{R}^{2}$ denote the closed disc of radius $r$ and center $(r, 0)$. Let

$$
X=\left\{(x, y, 1+r) \in \mathbb{R}^{3} \mid(x, y) \in D_{r} \text { and } 0<r<1\right\} .
$$

Let $\varphi: X \rightarrow \mathbb{R}^{3}$ be the inclusion map and define $\Psi$ and $\psi$ as in Lemma 6.4. Then $\Psi$ is convex (by Lemma 6.1), but it is not open as a map to its image, and neither is $\psi$. This shows that, in Lemma 6.4, convexity of the map $\left.\Psi\right|_{U}$ does not imply that the map $\left.\psi\right|_{V}$ is open to its image. Compare with Remark 8.2. 
6.7 Lemma Let $C$ be a subset of $\mathbb{R}^{n}$ such that $\mathbb{R}_{>0} \cdot C=C$. Let $U$ be a Hausdorff space and $\Psi: U \rightarrow C$ a continuous nonvanishing open map. Then $\bar{\Psi}:=\Psi /\|\Psi\|: U \rightarrow$ $C \cap S^{n-1}$ is an open map.

Proof The map $\bar{\Psi}$ is the composition of three maps: the map $\Psi$ from $U$ to $C \backslash\{0\}$, the map $w \mapsto(\|w\|, w /\|w\|)$ from $C \backslash\{0\}$ to $\mathbb{R}_{>0} \times\left(C \cap S^{n-1}\right)$, and the projection map $\mathbb{R}_{>0} \times\left(C \cap S^{n-1}\right) \rightarrow C \cap S^{n-1}$. The first of these maps is open by assumption; the second is open because it is a homeomorphism; the third is open by the definition of the product topology. Being the composition of three open maps, $\bar{\Psi}$ is open.

6.8 Proposition Let $X$ be a Hausdorff topological space and $\varphi: X \rightarrow \mathbb{R}^{n}$ a nonvanishing continuous map. Define maps $\Psi: X \times \mathbb{R}_{>0} \rightarrow \mathbb{R}^{n}$ and $\bar{\Psi}: X \times \mathbb{R}_{>0} \rightarrow S^{n-1}$ by $\Psi(x, \lambda)=\lambda \varphi(x)$ and $\bar{\Psi}=\Psi /\|\Psi\|$.

Assume that each point in $X \times \mathbb{R}_{>0}$ has a neighborhood $U$ such that the map $\left.\Psi\right|_{U}: U \rightarrow$ $\mathbb{R}^{n}$ is convex and such that the map $\left.\bar{\Psi}\right|_{U}: U \rightarrow \bar{\Psi}(U) \subset S^{n-1}$ is open to its image.

Assume that $X$ is compact and connected; assume that the image of the map $\varphi$ is not contained in a two dimensional subspace of $\mathbb{R}^{n}$.

Then, for every two points $y_{0}$ and $y_{1}$ in $X \times \mathbb{R}_{>0}$, if the segment $\left[\Psi\left(y_{0}\right), \Psi\left(y_{1}\right)\right]$ does not contain the origin, there exists a path $\gamma:[0,1] \rightarrow X \times \mathbb{R}_{>0}$ such that $\gamma(0)=y_{0}$, $\gamma(1)=y_{1}$, and $\Psi \circ \gamma$ is weakly monotone straight. Also, the map $\Psi$ is open as a map to its image.

\section{Proof Let}

$$
\psi=\varphi /\|\varphi\|: X \rightarrow S^{n-1} .
$$

By Corollary 6.5, every point in $X$ has a neighborhood $V$ such that the map $\left.\psi\right|_{V}$ is spherically convex and is open to its image.

Because the image of $\varphi$ is not contained in a two dimensional subspace of $\mathbb{R}^{n}$, the image of $\psi$ is not contained in a great circle.

Let $y_{0}=\left(x_{0}, \lambda_{0}\right)$ and $y_{1}=\left(x_{1}, \lambda_{1}\right)$ be two points in $X \times \mathbb{R}_{>0}$ such that the segment $\left[\Psi\left(y_{0}\right), \Psi\left(y_{1}\right)\right]$ does not contain the origin. Then $x_{0}$ and $x_{1}$ are points in $X$ such that $\psi\left(x_{1}\right) \neq-\psi\left(x_{0}\right)$.

By Corollary 5.2, there exists a path $x(t), 0 \leq t \leq 1$, such that $x(0)=x_{0}$ and $x(1)=x_{1}$, and such that $\psi(x(t))$ is a weakly monotone short geodesic.

By Lemma 3.4 there exists a weakly monotone straight path

$$
\bar{\gamma}:[0,1] \rightarrow \mathbb{R}^{n}
$$


such that $\bar{\gamma}(0)=\lambda_{0} \varphi\left(x_{0}\right), \bar{\gamma}(1)=\lambda_{1} \varphi\left(x_{1}\right)$, and $\bar{\gamma}(t) /\|\bar{\gamma}(t)\|=\psi(x(t))$. Then $\bar{\gamma}(t)=\lambda(t) \varphi(x(t))$, where $\lambda(t)=\|\bar{\gamma}(t)\| /\|\varphi(x(t))\|$. The path $\gamma(t):=(x(t), \lambda(t))$ in $X \times \mathbb{R}_{>0}$ satisfies $\gamma(0)=y_{0}, \gamma(1)=y_{1}$, and $\Psi(\gamma(\cdot))=\bar{\gamma}(\cdot)$ is weakly monotone straight.

By Corollary 5.2, the map $\psi: X \rightarrow S^{n-1}$ is open as a map to its image. From this it follows that the map

$$
X \times \mathbb{R}_{>0} \rightarrow S^{n-1} \times \mathbb{R}_{>0}, \quad(x, \lambda) \mapsto(\psi(x), \lambda)
$$

is open as a map to its image. From the commuting diagram

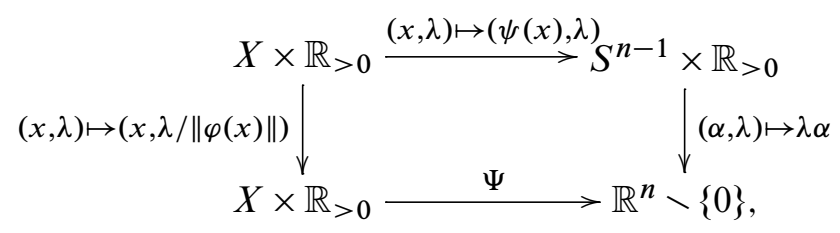

in which the vertical arrows are homeomorphisms and the top arrow is open to its image, it follows that $\Psi$ is open to its image.

6.10 Example Let $\varphi: S^{n-1} \rightarrow \mathbb{R}^{n}$ be the inclusion map. Then the map

$$
\Psi: S^{n-1} \times \mathbb{R}_{>0} \rightarrow \mathbb{R}^{n}, \quad(x, \lambda) \mapsto \lambda x,
$$

satisfies the assumptions and the conclusion of Proposition 6.8. Note that the map $\Psi$ itself is not convex.

6.11 Example Let $\varphi:[-\pi, \pi] \rightarrow \mathbb{R}^{2}$ be the map

$$
\varphi(t)=(\cos t, \sin t) .
$$

Then the map

$$
\Psi:[-\pi, \pi] \times \mathbb{R}_{>0} \rightarrow \mathbb{R}^{2}, \quad(t, \lambda) \mapsto(\lambda \cos t, \lambda \sin t)
$$

is not open as a map to its image, $\mathbb{R}^{2} \backslash\{0\}$, although every point has a neighborhood on which the map is convex and is open as a map to its image. Also, if $\left[t_{0}, t_{1}\right] \subset$ $[-\pi, \pi]$ is a subinterval of length $>\pi$, then, for any $\lambda_{0}, \lambda_{1} \in \mathbb{R}_{>0}$, the segment $\left[\Psi\left(t_{0}, \lambda_{0}\right), \Psi\left(t_{1}, \lambda_{1}\right)\right]$ does not contain the origin, but the points $\left(t_{0}, \lambda_{0}\right)$ and $\left(t_{1}, \lambda_{1}\right)$ cannot be connected by a path in $[-\pi, \pi] \times \mathbb{R}_{>0}$ whose image under $\Psi$ is this segment. Thus, the conclusions of Proposition 6.8 do not always hold if we allow the image of $\varphi$ to be contained in a two dimensional space. 


\section{Excision of a neighborhood of zero}

7.1 Remark We repeatedly use the following properties of a continuous map that is open as a map to its image:

(1) The restriction of this map to an open subset is open as a map to its image.

(2) The restriction of this map to the preimage of any set is also open to its image.

Thus, given a map $\varphi: U \rightarrow \mathbb{R}^{n}$, if $\varphi$ is open as a map to its image, then, for any open subset $V \subset U$, the map $\left.\varphi\right|_{\varphi^{-1}\left(\partial B_{\delta}\right) \cap V}$ is open as a map to its image.

7.2 Lemma Let $X$ be a Hausdorff topological space and $\varphi: X \rightarrow \mathbb{R}^{n}$ a continuous proper map. Suppose that for every point $x$ of $\varphi^{-1}(0)$ there exists an open neighborhood $U_{x}$ of $x$ in $X$ and a closed convex cone $C_{x}$ in $\mathbb{R}^{n}$ with vertex at the origin such that:

- The cone $C_{x}$ is not contained in a two dimensional subspace of $\mathbb{R}^{n}$.

- The image $\varphi\left(U_{x}\right)$ is an open subset of $C_{x}$.

- The map $\left.\varphi\right|_{U_{x}}: U_{x} \rightarrow \varphi\left(U_{x}\right)$ is open and convex and, for sufficiently small $\delta>0$, every point in $\varphi^{-1}\left(\partial B_{\delta}\right) \cap U_{x}$ has a neighborhood $V \subset U_{x}$ such that the restriction $\left.\varphi\right|_{\varphi^{-1}\left(\partial B_{\delta}\right) \cap V}$ is spherically convex.

Then there exist open subsets $W_{1}, \ldots, W_{N}$ of $X$ and closed convex cones $C_{1}, \ldots, C_{N}$ with vertex at the origin and there exists $\varepsilon>0$ such that:

- The sets $W_{1}, \ldots, W_{N}$ are disjoint and their union is equal to $\varphi^{-1}\left(B_{\varepsilon}\right)$.

- For each $i$, the image $\varphi\left(W_{i}\right)$ is equal to $C_{i} \cap B_{\varepsilon}$.

- For each $i$, the map $\left.\varphi\right|_{W_{i}}: W_{i} \rightarrow \varphi\left(W_{i}\right)$ is open and convex.

- For each $i$ and each $0<\delta<\varepsilon$, the restriction $\left.\varphi\right|_{\varphi^{-1}\left(\partial B_{\delta}\right) \cap W_{i}}$ is spherically convex, and its image is not equal to a pair of antipodal points.

Proof Because the level set $\varphi^{-1}(0)$ is compact and locally connected, it has finitely many connected components, $\left[x_{1}\right], \ldots,\left[x_{N}\right]$.

For each $i=1, \ldots, N$, by applying Lemma 4.4 to the set $K=\left[x_{i}\right]$, we choose an open subset $U_{i}$ of $X$ and a closed convex cone $C_{i}$ with vertex at the origin such that:

(a) The set $U_{i}$ contains the component $\left[x_{i}\right]$ of $\varphi^{-1}(0)$.

(b) The cone $C_{i}$ is not contained in a two dimensional subspace of $\mathbb{R}^{n}$.

(c) The image $\varphi\left(U_{i}\right)$ is an open subset of $C_{i}$. 
(d) The map $\left.\varphi\right|_{U_{i}}: U_{i} \rightarrow \varphi\left(U_{i}\right)$ is open and convex, and, for sufficiently small $\delta>0$, every point in $\varphi^{-1}\left(\partial B_{\delta}\right) \cap U_{i}$ has a neighborhood $U \subset U_{i}$ such that $\left.\varphi\right|_{\varphi^{-1}\left(\partial B_{\delta}\right) \cap U}$ is spherically convex.

We proceed in analogy with the proofs of Lemma 4.7 and of [9, Proposition 17]. Choose disjoint open subsets $\mathcal{O}_{1}, \ldots, \mathcal{O}_{N}$ of $X$ such that $\mathcal{O}_{i}$ contains $\left[x_{i}\right]$ and is contained in $U_{i}$; this is possible because $\varphi^{-1}(0)$ is compact and $X$ is Hausdorff.

By properties (c) and (d), there exist $\varepsilon_{i}>0$ such that $\varphi\left(U_{i}\right) \cap B_{\varepsilon_{i}}=C_{i} \cap B_{\varepsilon_{i}}$ and such that, if $0<\delta<\varepsilon_{i}$, every point in $\varphi^{-1}\left(\partial B_{\delta}\right) \cap U_{i}$ has a neighborhood $U \subset U_{i}$ such that $\left.\varphi\right|_{\varphi^{-1}\left(\partial B_{\delta}\right) \cap U}$ is spherically convex.

Choose $\varepsilon>0$ smaller than $\varepsilon_{1}, \ldots, \varepsilon_{N}$ and such that the preimage $\varphi^{-1}\left(B_{\varepsilon}\right)$ is contained in $\mathcal{O}_{1} \cup \cdots \cup \mathcal{O}_{N}$; this is possible because $\varphi$ is proper. Then every connected set that meets $\left[x_{i}\right]$ and is contained in $\varphi^{-1}\left(B_{\varepsilon}\right)$ must be contained in $\mathcal{O}_{i}$.

Set $W_{i}=U_{i} \cap \varphi^{-1}\left(B_{\varepsilon}\right)$. Because $\left.\varphi\right|_{U_{i}}$ is convex, so is $\left.\varphi\right|_{W_{i}}$; in particular, $W_{i}$ is connected. Because $W_{i}$ meets $\left[x_{i}\right]$ and is contained in $\varphi^{-1}\left(B_{\varepsilon}\right)$ it must be contained in $\mathcal{O}_{i}$. So $W_{1}, \ldots, W_{N}$ are disjoint, their union is $\varphi^{-1}\left(B_{\varepsilon}\right)$, and $\left.\varphi\right|_{W_{i}}: W_{i} \rightarrow C_{i}$ is convex and open.

Fix $\delta$ such that $0<\delta<\varepsilon$. The sets $W_{i} \cap \varphi^{-1}\left(\partial B_{\delta}\right)$ are open in $\varphi^{-1}\left(\partial B_{\delta}\right)$, disjoint, and they cover $\varphi^{-1}\left(\partial B_{\delta}\right)$. So each of these sets is closed in $\varphi^{-1}\left(\partial B_{\delta}\right)$, hence compact. Let $Y$ be a connected component of $\varphi^{-1}\left(\partial B_{\delta}\right) \cap W_{i}$. By property (d), every point in $Y$ has a neighborhood $U$ such that $\left.\varphi\right|_{Y \cap U}$ is spherically convex; it is also open as a map to its image, because $\left.\varphi\right|_{W_{i}}$ is. By properties (b) and (c), the image $\varphi(Y)$ is not contained in a great circle of $\partial B_{\delta}$. By Corollary 5.2, it follows that the map $\left.\varphi\right|_{Y}$ itself is spherically convex.

Because $\varphi(Y)$ is open and closed in $\partial B_{\delta} \cap C_{i}$, it is equal to $\partial B_{\delta} \cap C_{i}$. Because $\left.\varphi\right|_{W_{i}}$ has connected level sets, it follows that the connected component $Y$ is equal to the entire space $\varphi^{-1}\left(\partial B_{\delta}\right) \cap W_{i}$. Thus, the map $\left.\varphi\right|_{\varphi^{-1}\left(\partial B_{\delta}\right) \cap W_{i}}$ is spherically convex and its image is not equal to a pair of antipodal points.

7.3 Lemma Let $X$ be a Hausdorff topological space, $n \geq 2$, and $\varphi: X \rightarrow \mathbb{R}^{n}$ a continuous map. Let $\varepsilon>0$. Suppose that there exist open subsets $W_{1}, \ldots, W_{N}$ of $X$ such that:

- The sets $W_{1}, \ldots, W_{N}$ are disjoint and their union contains $\varphi^{-1}\left(\bar{B}_{\varepsilon}\right)$.

- For each $i$, the restriction $\left.\varphi\right|_{\varphi^{-1}\left(\partial B_{\varepsilon}\right) \cap W_{i}}$ is a spherically convex map, and its image is not equal to a pair of antipodal points.

Suppose that $X$ is path connected. Then the excised space $X^{\prime}:=X \backslash \varphi^{-1}\left(B_{\varepsilon}\right)$ is also path connected. 
Proof Denote $W_{0}:=X \backslash \varphi^{-1}\left(\bar{B}_{\varepsilon}\right)$. Then $W_{0}, W_{1}, \ldots, W_{N}$ is an open covering of $X$.

Let $x_{0}$ and $x_{1}$ be two points in $X^{\prime}$. Let $\gamma:[0,1] \rightarrow X$ be a path such that $\gamma(0)=x_{0}$ and $\gamma(1)=x_{1}$. Every $t \in[0,1]$ has a neighborhood $J$ in $[0,1]$ such that $\gamma(J)$ is entirely contained in one of the sets $W_{0}, W_{1}, \ldots, W_{N}$; we may assume that $J$ is an interval. Because the interval $[0,1]$ is compact, there exists a partition $0=t_{0}<t_{1}<\ldots<t_{M}=1$, and, for each $1 \leq j \leq M$, an integer $i_{j} \in\{0, \ldots, N\}$ such that the image $\gamma\left(\left[t_{j-1}, t_{j}\right]\right)$ is contained in the set $W_{i_{j}}$.

After possibly passing to a coarser partition of $[0,1]$, we assume that no two consecutive sets in the sequence $W_{i_{1}}, \ldots, W_{i_{M}}$ are equal. Also, because any two consecutive sets in this sequence meet at a division point $\gamma\left(t_{j}\right)$ whereas the sets $W_{1}, \ldots, W_{N}$ are disjoint, of any two consecutive sets in the sequence at least one must be $W_{0}$. So, because $W_{0}$ is contained in $X^{\prime}$, the interior division points $\gamma\left(t_{1}\right), \ldots, \gamma\left(t_{M-1}\right)$ must all be in $X^{\prime}$. By assumption, the endpoints $\gamma\left(t_{0}\right)=x_{0}$ and $\gamma\left(t_{M}\right)=x_{1}$ are also in $X^{\prime}$.

We now concentrate on the $j$-th subinterval, $\left[t_{j-1}, t_{j}\right]$. If $\gamma(t) \in X^{\prime}$ for all $t \in\left[t_{j-1}, t_{j}\right]$, then we define $\gamma_{j}:\left[t_{j-1}, t_{j}\right] \rightarrow X^{\prime}$ to be the restriction $\left.\gamma\right|_{\left[t_{j-1}, t_{j}\right]}$.

Otherwise, let $a$ and $b$ to be the infimum and supremum of the set $\left\{t \in\left[t_{j-1}, t_{j}\right] \mid\right.$ $\left.\gamma(t) \notin X^{\prime}\right\}$. So $[a, b] \subset\left[t_{j-1}, t_{j}\right], \gamma(a) \in \varphi^{-1}\left(\partial B_{\varepsilon}\right), \gamma(b) \in \varphi^{-1}\left(\partial B_{\varepsilon}\right)$, and $\gamma(t) \in X^{\prime}$ for $t_{j-1} \leq t \leq a$ and for $b \leq t \leq t_{j}$. Because the image $\gamma\left(\left[t_{j-1}, t_{j}\right]\right)$ is not contained in $X^{\prime}$, it must be contained in one of the sets $W_{1}, \ldots W_{N}$, say, in $W_{i}$. Because the restriction $\left.\varphi\right|_{\varphi^{-1}\left(\partial B_{\varepsilon}\right) \cap W_{i}}$ is spherically convex and its image is not equal to a pair of antipodal points, the set $\varphi^{-1}\left(\partial B_{\varepsilon}\right) \cap W_{i}$ is path connected. So there exists a path $\tilde{\gamma}:[a, b] \rightarrow \varphi^{-1}\left(\partial B_{\varepsilon}\right)$ connecting $\gamma(a)$ and $\gamma(b)$. Define

$$
\gamma_{j}(t)= \begin{cases}\gamma(t) & t_{j-1} \leq t \leq a, \\ \tilde{\gamma}(t) & a \leq t \leq b, \\ \gamma(t) & b \leq t \leq t_{j} .\end{cases}
$$

The concatenation of the paths $\gamma_{1}, \ldots, \gamma_{M}$ lies entirely in $X^{\prime}$ and it connects $x_{0}$ to $x_{1}$.

7.4 Lemma Let $W$ be a Hausdorff topological space. Let $C$ be a subset of $\mathbb{R}^{n}$ such that $\mathbb{R}_{>0} \cdot C=C$. Let $\varphi: W \rightarrow C$ be a continuous open map. Define maps $\Psi: W \times \mathbb{R}_{>0} \rightarrow C$ and $\bar{\Psi}:\left(W \backslash \varphi^{-1}(0)\right) \times \mathbb{R}_{>0} \rightarrow S^{n-1} \cap C$ by $\Psi(x, \lambda)=\lambda \varphi(x)$ and $\bar{\Psi}=\Psi /\|\Psi\|$.

Let $0<\varepsilon<\varepsilon^{\prime}$ be positive numbers. Suppose that the image of $\varphi$ contains $B_{\mathcal{\varepsilon}^{\prime}} \cap C$. Let

$$
W^{\prime}=W \backslash \varphi^{-1}\left(B_{\varepsilon}\right) .
$$


Then:

- The map $\left.\Psi\right|_{W^{\prime} \times \mathbb{R}_{>0}}: W^{\prime} \times \mathbb{R}_{>0} \rightarrow C$ is open and its image is $C \backslash\{0\}$.

- The map $\left.\bar{\Psi}\right|_{W^{\prime} \times \mathbb{R}_{>0}}: W^{\prime} \times \mathbb{R}_{>0} \rightarrow S^{n-1} \cap C$ is open.

Note that, above, $C$ is not necessarily closed.

Proof We begin with three consequences of the condition $\mathbb{R}_{>0} \cdot C=C$.

First, we identify the image of the map $\left.\Psi\right|_{W^{\prime} \times \mathbb{R}_{>0}}$. This image is equal to $\mathbb{R}_{>0}$. $\left(\varphi(W) \backslash B_{\varepsilon}\right)$, which is contained in the set $\mathbb{R}_{>0} \cdot\left(C \backslash B_{\varepsilon}\right)$ and contains the set $\mathbb{R}_{>0} \cdot\left(C \cap\left(B_{\mathcal{\varepsilon}^{\prime}} \backslash B_{\varepsilon}\right)\right)$; both of these sets are equal to $C \backslash\{0\}$. So the image of $\left.\Psi\right|_{W^{\prime} \times \mathbb{R}_{>0}}$ is $C \backslash\{0\}$ as claimed.

Next, we note that if $I$ is an open interval contained in $\mathbb{R}_{>0}$ and $\mathcal{O}$ is open in $C$, then the sets $I \cdot \mathcal{O}$ and $I \cdot\left(\mathcal{O} \cap \partial B_{\varepsilon}\right)$ are open in $C$.

Finally, suppose that $\Psi: W^{\prime} \times \mathbb{R}_{>0} \rightarrow C$ is open. Then $\Psi$ is also open as a map to $C \backslash\{0\}$. Since the central projection $\pi: \mathbb{R}^{n} \backslash\{0\} \rightarrow S^{n-1}$ given by $\pi(y)=y /\|y\|$ is open, and since $\pi^{-1}\left(S^{n-1} \cap C\right)=C \backslash\{0\}$, it follows that $\bar{\Psi}=\pi \circ \Psi: W^{\prime} \times \mathbb{R}_{>0} \rightarrow$ $S^{n-1} \cap C$ is also open, as a composition of two open maps.

It remains to show that the map $\Psi: W^{\prime} \times \mathbb{R}_{>0} \rightarrow C$ is open.

The sets of the form $Y^{\prime} \times I$, where $I$ is an open interval contained in $\mathbb{R}_{>0}$, and where $Y$ is an open subset of $W$ and $Y^{\prime}=Y \backslash \varphi^{-1}\left(B_{\varepsilon}\right)$, form a basis to the topology of $W^{\prime} \times \mathbb{R}_{>0}$. So we need to show that for every such set the image

$$
\Psi\left(Y^{\prime} \times I\right)=I \cdot\left(\varphi(Y) \backslash B_{\varepsilon}\right)
$$

is open in $C$. Let $\mu$ be a point in $I \cdot\left(\varphi(Y) \backslash B_{\varepsilon}\right)$, say, $\mu=\lambda \cdot \varphi\left(y^{\prime}\right)$ with $y^{\prime} \in$ $Y \backslash \varphi^{-1}\left(B_{\varepsilon}\right)$ and $\lambda \in I$.

If $y^{\prime}$ is actually in $Y \backslash \varphi^{-1}\left(\bar{B}_{\varepsilon}\right)$, then $\varphi(Y) \backslash \bar{B}_{\varepsilon}$ is an open neighborhood of $\varphi\left(y^{\prime}\right)$ in $C$, because $\varphi(Y)$ is open in $C$. It follows that $I \cdot\left(\varphi(Y) \backslash \bar{B}_{\varepsilon}\right)$ is an open neighborhood of $\lambda \cdot \varphi\left(y^{\prime}\right)$ in $C$.

Now suppose that $y^{\prime} \in \varphi^{-1}\left(\partial B_{\varepsilon}\right)$. Because $\varphi(Y)$ is open in $C$, its intersection with $\partial B_{\varepsilon}$ is open in $C \cap \partial B_{\varepsilon}$. It follows that the set $I \cdot\left(\varphi(Y) \cap \partial B_{\varepsilon}\right)$ is an open neighborhood of $\lambda \cdot \varphi\left(y^{\prime}\right)$ in $C$.

In either case, we found an open neighborhood of $\mu$ in $C$ that is contained in $I$. $\left(\varphi(Y) \backslash B_{\varepsilon}\right)$. This completes the proof of the lemma. 
7.5 Lemma Let $W$ be a Hausdorff topological space, let $C$ be a closed convex cone in $\mathbb{R}^{n}$ with vertex at the origin, and let $\varphi: W \rightarrow C$ be a continuous map. Define maps $\Psi: W \times \mathbb{R}_{>0} \rightarrow C$ and $\bar{\Psi}:\left(W \backslash \varphi^{-1}(0)\right) \times \mathbb{R}_{>0} \rightarrow S^{n-1} \cap C$ by $\Psi(x, \lambda)=\lambda \varphi(x)$ and $\bar{\Psi}=\Psi /\|\Psi\|$. Let $0<\varepsilon<\varepsilon^{\prime}$ be positive numbers. Let

$$
W^{\prime}=W \backslash \varphi^{-1}\left(B_{\varepsilon}\right) \text {. }
$$

Assume that:

- $\varphi(W)=B_{\mathcal{\varepsilon}^{\prime}} \cap C$.

- The map $\varphi$ is convex and open to its image.

- The map $\left.\varphi\right|_{\varphi^{-1}\left(\partial \boldsymbol{B}_{\varepsilon}\right)}$ is spherically convex.

Then every point in $W^{\prime} \times \mathbb{R}_{>0}$ has a neighborhood $U^{\prime}$ in $W^{\prime} \times \mathbb{R}_{>0}$ such that the map $\left.\Psi\right|_{U^{\prime}}$ is convex and the map $\left.\bar{\Psi}\right|_{U^{\prime}}$ is open to its image.

Proof Let $H$ be an open half-space in $\mathbb{R}^{n}$ whose boundary contains the origin, and let

$$
U^{\prime}=\left(\varphi^{-1}(H) \backslash \varphi^{-1}\left(B_{\varepsilon}\right)\right) \times \mathbb{R}_{>0} .
$$

We will prove that the map $\left.\Psi\right|_{U^{\prime}}$ is convex and that the map $\left.\bar{\Psi}\right|_{U^{\prime}}$ is open to its image. This will be enough, because the sets $U^{\prime}$, for different choices of $H$, form an open covering of $W^{\prime} \times \mathbb{R}_{>0}$.

By Lemma 7.4 applied to $\varphi: \varphi^{-1}(H) \rightarrow H \cap C$, the map $\left.\bar{\Psi}\right|_{U^{\prime}}$ is open as a map to its image. It remains to show that the map $\left.\Psi\right|_{U^{\prime}}$ is convex. This will overlap the proof of Lemma 7.3, in that we will connect two points of $W^{\prime}$ by a path that lies entirely in $W^{\prime}$, but here we must take care to obtain a path whose composition with $\varphi$ can be "straightened" by multiplication by a positive function.

Let

$$
U=\varphi^{-1}(H) \times \mathbb{R}_{>0} .
$$

Because $\varphi$ is a convex map and $H$ is a convex set, the restriction $\left.\varphi\right|_{\varphi^{-1}(H)}$ is a convex map. It follows by Lemma 6.1 that $\left.\Psi\right|_{U}$ is a convex map.

Let $y_{0}=\left(x_{0}, \lambda_{0}\right)$ and $y_{1}=\left(x_{1}, \lambda_{1}\right)$ be two points in $U^{\prime}$. The segment $\left[\Psi\left(y_{0}\right), \Psi\left(y_{1}\right)\right]$ is contained in $H$, so it does not pass through the origin. Because $\left.\Psi\right|_{U}$ is convex, there exists a path $y(t)=(x(t), \lambda(t))$ in $U$ connecting $y_{0}$ and $y_{1}$ with $\Psi \circ y$ weakly monotone straight. By Lemma 3.2, the path $\bar{\Psi}(y(\cdot))$ is a weakly monotone short geodesic.

Let

$$
\psi:=\varphi /\|\varphi\|: W \backslash \varphi^{-1}(0) \rightarrow S^{n-1} .
$$

Then

$$
\bar{\Psi}(x, \lambda)=\psi(x),
$$

and in particular $\bar{\Psi}(y(t))=\psi(x(t))$. So $\psi(x(\cdot))$ is a weakly monotone short geodesic. 
Since $x_{0}$ and $x_{1}$ are in $W^{\prime}$, the path $\varphi(x(\cdot))$ starts and ends outside $B_{\varepsilon}$. If the path $\varphi(x(\cdot))$ happens to lie entirely outside $B_{\varepsilon}$, then the path $y(\cdot)$ lies entirely in $U^{\prime}$, and we are done. Otherwise, let $[a, b] \subset[0,1]$ be such that $\varphi(x(a)) \in \partial B_{\varepsilon}, \varphi(x(b)) \in \partial B_{\varepsilon}$, and $\varphi(x(t)) \notin B_{\varepsilon}$ for $0 \leq t \leq a$ and for $b \leq t \leq 1$. For instance, we may take $a$ and $b$ to be the infimum and supremum of the set of times $t$ at which $\varphi(x(t))$ is in the ball $B_{\varepsilon}$.

Because $\left.\varphi\right|_{\varphi^{-1}\left(\partial \boldsymbol{B}_{\varepsilon} \cap H\right)}$ is spherically convex (cf Remark 4.3) and its image is not equal to a pair of antipodal points, there exists a path $\tilde{x}(t)$, for $a \leq t \leq b$, connecting $x(a)$ and $x(b)$ and lying in $\varphi^{-1}\left(\partial B_{\varepsilon} \cap H\right)$, such that $\varphi(\tilde{x}(t))$ is a weakly monotone short geodesic. Necessarily, $\tilde{x}(t)$ is in $W^{\prime}$. We define $\tilde{x}(t)$ to be equal to $x(t)$ on the segments $[0, a]$ and $[b, 1]$. Then $\psi(\tilde{x}(t))$ is a path in $S^{n-1}$ whose restriction to each of the segments $[0, a],[a, b]$, and $[b, 1]$ is a weakly monotone short geodesic. But the value of this path at the points $0, a, b, 1$ coincide with the values of $\psi(x(t))$, which is a weakly monotone short geodesic on the entire segment $[0,1]$. It follows that $\psi(\tilde{x}(t))$ is also a weakly monotone short geodesic on the entire segment $[0,1]$.

Let $\lambda_{0}^{\prime}=\left\|\Psi\left(y_{0}\right)\right\|$ and $\lambda_{1}^{\prime}=\left\|\Psi\left(y_{1}\right)\right\|$. Then $\Psi\left(y_{0}\right)=\lambda_{0}^{\prime} \psi(\tilde{x}(0))$ and $\Psi\left(y_{1}\right)=$ $\lambda_{1}^{\prime} \psi(\tilde{x}(1))$. Lemma 3.4 implies that there exists a weakly monotone straight path $\bar{\gamma}:[0,1] \rightarrow \mathbb{R}^{n}$ from $\Psi\left(y_{0}\right)$ to $\Psi\left(y_{1}\right)$ such that $\bar{\gamma} /\|\bar{\gamma}\|=\psi(\tilde{x}(\cdot))$. In particular, $\bar{\gamma}(t)$ is a positive multiple of $\varphi(\tilde{x}(t))$. So there exists a continuous function $\tilde{\lambda}:[0,1] \rightarrow$ $\mathbb{R}_{>0}$ such that $\bar{\gamma}(t)=\tilde{\lambda}(t) \varphi(\tilde{x}(t))$; namely, $\tilde{\lambda}(t)=\|\bar{\gamma}(t)\| /\|\varphi(\tilde{x}(t))\|$. So $\tilde{y}(\cdot)=$ $(\tilde{x}(\cdot), \tilde{\lambda}(\cdot))$ is a path in $U^{\prime}$ from $y_{0}$ to $y_{1}$ such that $\Psi \circ y$ is weakly monotone straight.

7.6 Lemma Let $X$ be a compact connected Hausdorff topological space and $\varphi: X \rightarrow$ $\mathbb{R}^{n}$ a continuous map. Define maps $\Psi: X \times \mathbb{R}_{>0} \rightarrow \mathbb{R}^{n}$ and $\bar{\Psi}:\left(X \backslash \varphi^{-1}(0)\right) \times \mathbb{R}_{>0} \rightarrow$ $S^{n-1}$ by $\Psi(x, \lambda)=\lambda \varphi(x)$ and $\bar{\Psi}=\Psi /\|\Psi\|$. Assume that:

- The image $\varphi(X)$ contains 0 .

- For every point $x$ of $X$ with $\varphi(x)=0$ there exists an open neighborhood $U_{x}$ of $x$ in $X$ and a closed convex cone $C_{x}$ in $\mathbb{R}^{n}$ with vertex at the origin such that:

- The cone $C_{x}$ is not contained in a two dimensional subspace of $\mathbb{R}^{n}$.

- The image $\varphi\left(U_{x}\right)$ is an open subset of $C_{x}$.

- The map $\left.\varphi\right|_{U_{x}}: U_{x} \rightarrow \varphi\left(U_{x}\right)$ is convex and open, and, for sufficiently small $\delta>0$, every point in $\varphi^{-1}\left(\partial B_{\delta}\right) \cap U_{x}$ has a neighborhood $V \subset U_{x}$ such that the restriction $\left.\varphi\right|_{\varphi^{-1}\left(\partial B_{\delta}\right) \cap V}$ is spherically convex.

- For every point $(x, \lambda)$ of $X \times \mathbb{R}_{>0}$ with $\varphi(x) \neq 0$, every neighborhood of $(x, \lambda)$ in $X \times \mathbb{R}_{>0}$ contains a smaller neighborhood, $U$, such that the map $\left.\Psi\right|_{U}$ is convex and such that the map $\left.\bar{\Psi}\right|_{U}$ is open as a map to its image. 
Then, for every sufficiently small positive number $\varepsilon$, the following results hold. Let

$$
X^{\prime}=X \backslash \varphi^{-1}\left(B_{\varepsilon}\right) \text {. }
$$

Then:

(1) For every two points $y_{0}$ and $y_{1}$ in $X^{\prime} \times \mathbb{R}_{>0}$, if the segment $\left[\Psi\left(y_{0}\right), \Psi\left(y_{1}\right)\right]$ does not contain the origin, then there exists a path $\gamma:[0,1] \rightarrow X^{\prime} \times \mathbb{R}_{>0}$ such that $\gamma(0)=y_{0}, \gamma(1)=y_{1}$, and $\Psi \circ \gamma$ is weakly monotone straight.

(2) The map $\Psi: X^{\prime} \times \mathbb{R}_{>0} \rightarrow \Psi\left(X^{\prime} \times \mathbb{R}_{>0}\right)$ is open as a map to its image.

(3) The image $\Psi\left(X^{\prime} \times \mathbb{R}_{>0}\right)$ is equal to $\Psi\left(X \times \mathbb{R}_{>0}\right) \backslash\{0\}$.

Proof The space $X$ and the map $\varphi$ satisfy the assumptions of Lemma 7.2. Let $W_{1}, \ldots, W_{N}$ be open subsets of $X$, let $C_{1}, \ldots, C_{N}$ be closed convex cones with vertex at the origin, and let $\varepsilon^{\prime}$ be a positive number, such that:

- The sets $W_{1}, \ldots, W_{N}$ are disjoint and their union is equal to $\varphi^{-1}\left(B_{\varepsilon^{\prime}}\right)$.

- For each $i$, the image $\varphi\left(W_{i}\right)$ is equal to $C_{i} \cap B_{\varepsilon^{\prime}}$.

- For each $i$, the map $\left.\varphi\right|_{W_{i}}: W_{i} \rightarrow \varphi\left(W_{i}\right)$ is open and convex, and, for each $0<\varepsilon<\varepsilon^{\prime}$, the restriction $\left.\varphi\right|_{\varphi^{-1}\left(\partial B_{\varepsilon}\right) \cap W_{i}}$ is spherically convex and its image is not equal to a pair of antipodal points.

Let $\varepsilon$ be any positive number such that $0<\varepsilon<\varepsilon^{\prime}$, and let $X^{\prime}=X \backslash \varphi^{-1}\left(B_{\varepsilon}\right)$.

Because $X$ is connected and locally path connected, $X$ is path connected. The space $X$, the map $\varphi$ and the number $\varepsilon$ satisfy the assumptions of Lemma 7.3. Thus, the excised space $X^{\prime}$ is path connected.

For each $1 \leq i \leq N$, the set $W_{i}$, the cone $C_{i}$, the map $\left.\varphi\right|_{W_{i}}: W_{i} \rightarrow C_{i}$, and the numbers $\varepsilon^{\prime}$ and $\varepsilon$ satisfy the assumptions of Lemma 7.4. By the first part of that lemma,

$$
\Psi\left(\left(W_{i} \cap X^{\prime}\right) \times \mathbb{R}_{>0}\right)=\Psi\left(W_{i} \times \mathbb{R}_{>0}\right) \backslash\{0\} .
$$

Because $X=X^{\prime} \cup \bigcup_{i=1}^{N} W_{i}$, this implies that

$$
\Psi\left(X^{\prime} \times \mathbb{R}_{>0}\right)=\Psi\left(X \times \mathbb{R}_{>0}\right) \backslash\{0\},
$$

which is item (3) that we had set to prove.

By the assumptions on $\varphi^{-1}(0)$, the image $\varphi(X)$ contains a subset (namely, $\varphi\left(U_{x}\right)$ for $\varphi(x)=0$ ) that is not contained in any two dimensional subspace of $\mathbb{R}^{n}$. Rewriting (7.8) as $\mathbb{R}_{>0} \cdot \varphi\left(X^{\prime}\right)=\mathbb{R}_{>0} \cdot \varphi(X) \backslash\{0\}$, we deduce that $\varphi\left(X^{\prime}\right)$ is not contained in a two dimensional subspace of $\mathbb{R}^{n}$ either. 
Let $(x, \lambda)$ be a point of $X^{\prime} \times \mathbb{R}_{>0}$. If $x$ belongs to one of the sets $W_{1}, \ldots, W_{N}$, then Lemma 7.5 guarantees the existence of a neighborhood $U^{\prime}$ of $(x, \lambda)$ in $X^{\prime} \times \mathbb{R}_{>0}$ such that the map $\left.\Psi\right|_{U^{\prime}}$ is convex and the map $\left.\bar{\Psi}\right|_{U^{\prime}}$ is open to its image. If $x$ does not belong to any $W_{i}$, then $\varphi(x)$ is outside $B_{\varepsilon^{\prime}}$, and $\left(X \backslash \varphi^{-1}\left(\bar{B}_{\varepsilon}\right)\right) \times \mathbb{R}_{>0}$ is a neighborhood of $(x, \lambda)$ in $X \times \mathbb{R}_{>0}$; by assumption, it contains a smaller neighborhood, $U$, such that $\left.\Psi\right|_{U}$ is convex and $\left.\bar{\Psi}\right|_{U}$ is open to its image.

We have just shown that every point in $X^{\prime} \times \mathbb{R}_{>0}$ has a neighborhood $U^{\prime}$ in $X^{\prime} \times \mathbb{R}_{>0}$ such that the map $\left.\Psi\right|_{U^{\prime}}$ is convex and the map $\left.\bar{\Psi}\right|_{U^{\prime}}$ is open to its image. We have also shown that $X^{\prime}$ is connected and that $\varphi\left(X^{\prime}\right)$ is not contained in a two dimensional subspace of $\mathbb{R}^{n}$. Also, being a closed subset of a compact space, $X^{\prime}$ is compact. Thus, the space $X^{\prime}$ and the map $\left.\varphi\right|_{X^{\prime}}$ satisfy the assumptions of Proposition 6.8. The conclusions of this proposition are exactly the items (1) and (2) that we had set to prove.

7.9 Definition A continuous map $\varphi: X \rightarrow B$ has the weak path lifting property if, for any point $x$ in $X$ and path $\gamma:[0,1] \rightarrow B$ with $\gamma(0)=\varphi(x)$, there exists a path $\tilde{\gamma}:[0,1] \rightarrow X$ and a weakly monotone reparametrization $s:[0,1] \rightarrow[0,1]$, with $s(0)=0$ and $s(1)=1$, such that $\tilde{\gamma}(0)=x$ and $\varphi(\tilde{\gamma}(t))=\gamma(s(t))$ for all $t \in[0,1]$.

7.10 Lemma Let $U$ be a Hausdorff topological space. Let $C$ be a closed convex cone in $\mathbb{R}^{n}$ with vertex at the origin. Let $\varepsilon>0$. Let $\varphi: U \rightarrow C$ be a continuous map. Assume that:

- The image $\varphi(U)$ contains $B_{\varepsilon} \cap C$.

- The map $\varphi: U \rightarrow \varphi(U)$ has the weak path lifting property, and its level sets are path connected.

Then:

- The map $\left.\varphi\right|_{\varphi^{-1}\left(\boldsymbol{B}_{\varepsilon}\right)}$ is convex.

- For every $0<\delta<\varepsilon$, every point $x$ in $\varphi^{-1}\left(\partial B_{\delta}\right)$ has a neighborhood $V_{x}$ such that the map $\left.\varphi\right|_{\varphi^{-1}\left(\partial B_{\delta}\right) \cap V_{x}}$ is spherically convex.

Proof For any subset $E$ of $\mathbb{R}^{n}$, the restriction $\left.\varphi\right|_{\varphi^{-1}(E)}: \varphi^{-1}(E) \rightarrow E \cap \varphi(U)$ has the weak path lifting property, and its level sets are path connected. This follows from the analogous properties of $\varphi$. If the set $E \cap \varphi(U)$ is convex, it follows that the map $\left.\varphi\right|_{\varphi^{-1}(E)}$ is convex. If the set $E \cap \varphi(U)$ is contained in $\partial B_{\delta}$ and is spherically convex, it follows that the map $\left.\varphi\right|_{\varphi^{-1}(E)}: \varphi^{-1}(E) \rightarrow \partial B_{\delta}$ is spherically convex. 
The first part of the lemma follows by setting $E=B_{\varepsilon}$ and noting that the set $E \cap \varphi(U)=$ $B_{\varepsilon} \cap C$ is convex.

For the second part of the lemma, fix $0<\delta<\varepsilon$ and fix $x$ in $\varphi^{-1}\left(\partial B_{\delta}\right)$. Let $H_{x} \subset \mathbb{R}^{n}$ be an open half-space whose boundary contains the origin such that $\varphi(x) \in H_{x}$. Let $E=H_{x} \cap \partial B_{\delta}$. Then $E \cap \varphi(U)=\partial B_{\delta} \cap H_{x} \cap C$ is a spherically convex subset of $\partial B_{\delta}$, and $\varphi^{-1}(E)=\varphi^{-1}\left(\partial B_{\delta}\right) \cap \varphi^{-1}\left(H_{x}\right)$. So $V_{x}:=\varphi^{-1}\left(H_{x}\right)$ is a neighborhood of $x$ such that the map $\left.\varphi\right|_{\varphi^{-1}\left(\partial B_{\delta}\right) \cap V_{x}}$ is spherically convex.

7.11 Proposition Let $X$ be a compact connected Hausdorff topological space and $\varphi: X \rightarrow \mathbb{R}^{n}$ a continuous map. Define maps

$$
\Psi: X \times \mathbb{R}_{>0} \rightarrow \mathbb{R}^{n} \quad \text { and } \quad \bar{\Psi}:\left(X \backslash \varphi^{-1}(0)\right) \times \mathbb{R}_{>0} \rightarrow S^{n-1}
$$

by $\Psi(x, \lambda)=\lambda \varphi(x)$ and $\bar{\Psi}=\Psi /\|\Psi\|$. Assume that:

- The image $\varphi(X)$ contains 0 .

- For every point $x$ of $X$ with $\varphi(x)=0$, there exists an open neighborhood $U_{x}$ of $x$ in $X$ and a closed convex cone $C_{x}$ in $\mathbb{R}^{n}$ with vertex at the origin such that:

- The cone $C_{x}$ is not contained in a two dimensional subspace of $\mathbb{R}^{n}$.

- The image $\varphi\left(U_{x}\right)$ is an open subset of $C_{x}$.

- The map $\left.\varphi\right|_{U_{x}}: U_{x} \rightarrow \varphi\left(U_{x}\right)$ is open, has the weak path lifting property, and its level sets are path connected.

- For every point $(x, \lambda)$ of $X \times \mathbb{R}_{>0}$ with $\varphi(x) \neq 0$, every neighborhood of $(x, \lambda)$ in $X \times \mathbb{R}_{>0}$ contains a smaller neighborhood $U$ such that the map $\left.\Psi\right|_{U}$ is convex and such that the map $\left.\bar{\Psi}\right|_{U}$ is (defined and) open as a map to its image.

Then the following results hold. Let

$$
C=\Psi\left(X \times \mathbb{R}_{>0}\right)=\mathbb{R}_{>0} \cdot \varphi(X) .
$$

(1) For any two points $y_{0}$ and $y_{1}$ in $X \times \mathbb{R}_{>0}$, if $\Psi\left(y_{0}\right)$ and $\Psi\left(y_{1}\right)$ are not both zero, there exists a path $\gamma:[0,1] \rightarrow X \times \mathbb{R}_{>0}$ such that $\gamma(0)=y_{0}$ and $\gamma(1)=y_{1}$ and such that $\Psi \circ \gamma:[0,1] \rightarrow \mathbb{R}^{n}$ is weakly monotone straight.

(2) For every $x \in \varphi^{-1}(0)$, the cone $C_{x}$ is equal to $C$.

(3) The map $\Psi$ is open as a map to $C$.

Proof For $x \in \varphi^{-1}(0)$, that fact that the image $\varphi\left(U_{x}\right)$ is open in $C_{x}$ implies that $\varphi\left(U_{x}\right)$ contains $B_{\varepsilon} \cap C_{x}$ for some $\varepsilon>0$. Applying Lemma 7.10 to $\varphi: U_{x} \rightarrow C_{x}$, and after replacing $U_{x}$ by $\varphi^{-1}\left(B_{\varepsilon}\right) \cap U_{x}$ (and continuing to denote it by $U_{x}$ ), we get the following facts. 
- $\left.\varphi\right|_{U_{x}}: U_{x} \rightarrow C_{x}$ is convex and open.

- For every sufficiently small $\delta>0$, every point in $\varphi^{-1}\left(\partial B_{\delta}\right) \cap U_{x}$ has a neighborhood $V \subset U_{x}$ such that $\left.\varphi\right|_{\varphi^{-1}\left(\partial B_{\delta}\right) \cap V}$ is spherically convex.

The space $X$ and the map $\varphi$ now satisfy the assumptions of Lemma 7.6. Let $\varepsilon^{\prime}$ be a positive number that is sufficiently small so that the conclusions of Lemma 7.6 hold for all $\varepsilon$ such that $0<\varepsilon<\varepsilon^{\prime}$. Then, for every such $\varepsilon$, the following facts are true. Let $X_{\varepsilon}^{\prime}:=X \backslash \varphi^{-1}\left(B_{\varepsilon}\right)$.

(a) For every two points $y_{0}$ and $y_{1}$ in $X_{\varepsilon}^{\prime} \times \mathbb{R}_{>0}$, if the segment $\left[\Psi\left(y_{0}\right), \Psi\left(y_{1}\right)\right]$ does not contain the origin, then there exists a path in $X_{\varepsilon}^{\prime} \times \mathbb{R}_{>0}$ connecting $y_{0}$ to $y_{1}$ whose composition with $\Psi$ is weakly monotone straight.

(b) The image $\Psi\left(X_{\varepsilon}^{\prime} \times \mathbb{R}_{>0}\right)$ is equal to $C \backslash\{0\}$.

(c) The map $\left.\Psi\right|_{X_{\varepsilon}^{\prime} \times \mathbb{R}_{>0}}$ is open as a map to $C$.

Because the space $X \backslash \varphi^{-1}(0)$ is the union of the open subsets $X_{\varepsilon}^{\prime}$, for $0<\varepsilon<\varepsilon^{\prime}$, and by the fact (c), the map $\left.\Psi\right|_{\left(X \backslash \varphi^{-1}(0)\right) \times \mathbb{R}_{>0}}$ is open as a map to $C$.

Choose any $x \in \varphi^{-1}(0)$. Consider the set $\Psi\left(\left(U_{x} \backslash \varphi^{-1}(0)\right) \times \mathbb{R}_{>0}\right)$. This set is equal to $C_{x} \backslash\{0\}$, because $C_{x}$ is a cone with vertex at the origin and $\varphi\left(U_{x}\right)$ is open in $C_{x}$ and contains the origin. But this set is also open in $C$, because $\left.\Psi\right|_{\left(X \backslash \varphi^{-1}(0)\right) \times \mathbb{R}_{>0}}$ is open as a map to $C$. So $C_{x} \backslash\{0\}$ is open in $C$. Because $C_{X}$ is a closed convex cone and $C$ is connected, it follows that $C_{x}$ is equal to $C$. This proves Claim (2).

In particular, since $C_{x}=C$, we now know that the restriction of $\varphi$ to $U_{x}$ is open as a map to $C$. This implies that the restriction of $\Psi$ to $U_{x} \times \mathbb{R}_{>0}$ is also open as a map to $C$. This is true for every $x \in \varphi^{-1}(0)$. But we also showed that the restriction of $\Psi$ to $\left(X \backslash \varphi^{-1}(0)\right) \times \mathbb{R}_{>0}$ is open as a map to $C$. Because the space $X$ is the union of the open subsets $U_{x}$ for $x \in \varphi^{-1}(0)$ and the open subset $X \backslash \varphi^{-1}(0)$, we obtain Claim (3).

Claim (1), in the case that $0 \notin\left[\Psi\left(y_{0}\right), \Psi\left(y_{1}\right)\right]$, follows from the above fact (a) when $\varepsilon$ is chosen small enough so that both $y_{0}$ and $y_{1}$ lie in $X_{\varepsilon}^{\prime} \times \mathbb{R}_{>0}$.

Suppose that $\Psi\left(y_{0}\right)$ and $\Psi\left(y_{1}\right)$ are both nonzero but $0 \in\left[\Psi\left(y_{0}\right), \Psi\left(y_{1}\right)\right]$. Choose any $y^{\prime} \in \Psi^{-1}(0)$. If we can find paths from $y_{0}$ to $y^{\prime}$ and from $y^{\prime}$ to $y_{1}$ whose compositions with $\Psi$ are weakly monotone straight paths, their concatenation will give a path from $y_{0}$ to $y_{1}$ whose composition with $\Psi$ is a weakly monotone straight path.

It remains to prove Claim (1) in the case that, say, $\Psi\left(y_{0}\right)=0$ and $\Psi\left(y_{1}\right) \neq 0$. Fix such $y_{0}$ and $y_{1}$. Write $y_{0}=(x, \lambda)$; then $x$ is in $\varphi^{-1}(0)$. By the definition of $C$ 
and because $C=C_{x}$, the value $\Psi\left(y_{1}\right)$ belongs to $C_{x}$. Because $\varphi\left(U_{x}\right)$ is an open subset of $C_{x}$ that contains the origin, if $\varepsilon$ is a sufficiently small positive number, then $\varepsilon \Psi\left(y_{1}\right)$ belongs to $\varphi\left(U_{x}\right)$. Fix such an $\varepsilon$, and let $x^{\prime}$ be a point in $U_{x}$ such that $\varphi\left(x^{\prime}\right)=\varepsilon \Psi\left(y_{1}\right)$. Because $\left.\varphi\right|_{U_{x}}$ is convex, there exists a path $x(\cdot)$ in $U_{x}$ from $x$ to $x^{\prime}$ whose composition with $\varphi$ is a weakly monotone straight path from the origin to $\varepsilon \Psi\left(y_{1}\right)$.

Then $(x(\cdot), 1)$ is a path from $(x, 1)$ to $\left(x^{\prime}, 1\right)$ whose composition with $\Psi$ is a weakly monotone straight path from the origin to $\varepsilon \Psi\left(y_{1}\right)$. Concatenating with a path from $y_{0}=(x, \lambda)$ to $(x, 1)$ which is entirely contained in $\{x\} \times \mathbb{R}_{>0}$, we obtain a path $\gamma^{\prime}$ from $y_{0}$ to $\left(x^{\prime}, 1\right)$ whose composition with $\Psi$ is a weakly monotone straight path from the origin to $\varepsilon \Psi\left(y_{1}\right)$.

By the case of Claim (1) that we already proved, there exists a path $\gamma^{\prime \prime}$ from $\left(x^{\prime}, 1\right)$ to $y_{1}$ whose composition with $\Psi$ is a weakly monotone straight path from $\varepsilon \Psi\left(y_{1}\right)$ to $\Psi\left(y_{1}\right)$. The concatenation of $\gamma^{\prime}$ with $\gamma^{\prime \prime}$ is a path from $y_{0}$ to $y_{1}$ whose composition with $\Psi$ is weakly monotone straight.

This completes the proof of Claim (1) and of Proposition 7.11.

\section{Linear maps on the simplex}

We begin by setting some notation:

$$
\begin{aligned}
\mathbb{R}_{+}^{n} & =\left\{\left(s_{1}, \ldots, s_{n}\right) \in \mathbb{R}^{n} \mid s_{j} \geq 0 \text { for } j=1, \ldots, n\right\}, \\
\Lambda & =\left\{\left(s_{1}, \ldots, s_{n}\right) \in \mathbb{R}_{+}^{n} \mid s_{1}+\cdots+s_{n}<1\right\} .
\end{aligned}
$$

The goal of this section is to prove the following proposition.

8.1 Proposition Let $L: \mathbb{R}^{n} \rightarrow \mathbb{R}^{k}$ be a linear map, and let $A=L(\Lambda)$. Then the map

$$
\left.L\right|_{\Lambda}: \Lambda \rightarrow A
$$

is open, has the weak path lifting property (cf Definition 7.9), and its level sets are path connected.

8.2 Remark The restriction of a linear projection to a convex set is not necessarily open as a map to its image. For example, let $D_{r} \subset \mathbb{R}^{2}$ denote the closed disc of radius $r$ and center $(r, 0)$, and let $X=\left\{(x, y, r) \in \mathbb{R}^{3} \mid(x, y) \in D_{r}\right\}$. Then $X$ is convex, and the restriction to $X$ of the projection $(x, y, z) \mapsto(x, y)$ is not open as a map to its image. 
We will need the following variant of Carathéodory's theorem from convex geometry:

8.3 Lemma Let $v_{1}, \ldots, v_{n}$ be vectors in $\mathbb{R}^{k}$. Let $w=\sum s_{j} v_{j}$ with $\left(s_{1}, \ldots, s_{n}\right) \in \Lambda$. Then there exists $\left(s_{1}^{\prime}, \ldots, s_{n}^{\prime}\right) \in \Lambda$ such that $w=\sum s_{j}^{\prime} v_{j}$ and such that the vectors $v_{j}$ for which $s_{j}^{\prime} \neq 0$ are linearly independent.

Proof We prove the lemma by induction on $n$. If $n=1$, the lemma is obvious. Suppose that the lemma is true for $n-1$ vectors, and we will prove it for $n$ vectors.

Suppose that $w=\sum_{j=1}^{n} s_{j} v_{j}$ with $\left(s_{1}, \ldots, s_{n}\right) \in \Lambda$ and that $v_{1}, \ldots, v_{n}$ are not linearly independent. If one or more of $s_{1}, \ldots, s_{n}$ is zero, the required conclusion follows from the induction hypothesis. Suppose that $s_{1}, \ldots, s_{n}$ are all positive.

Then there exist $\lambda_{1}, \ldots, \lambda_{n}$ not all zero such that $\sum \lambda_{j} \geq 0$ and $\sum \lambda_{j} v_{j}=0$. At least one of the $\lambda_{j} \mathrm{~s}$ is positive. Choose $c=\min \left\{s_{j} / \lambda_{j} \mid \lambda_{j}>0\right\}=s_{i} / \lambda_{i}$. Then

$$
w=\sum s_{j} v_{j}-c \sum \lambda_{j} v_{j}=\sum\left(s_{j}-c \lambda_{j}\right) v_{j} .
$$

Note that $s_{j}-c \lambda_{j} \geq 0$ for all $j$, and $s_{i}-c \lambda_{i}=0$. Furthermore, since $c>0$ and $\sum \lambda_{j} \geq 0$

$$
\sum\left(s_{j}-c \lambda_{j}\right)=\sum s_{j}-c \sum \lambda_{j} \leq \sum s_{j}<1 .
$$

The required conclusion now follows from the induction hypothesis applied to the vectors $\left\{v_{1}, \ldots, v_{n}\right\} \backslash\left\{v_{i}\right\}$.

8.4 Lemma Let $L: \mathbb{R}^{n} \rightarrow \mathbb{R}^{k}$ be a linear map, and let $C=L\left(\mathbb{R}_{+}^{n}\right)$. Then $L(\Lambda)$ is a relative neighborhood of 0 in $C$.

Proof Let $e_{1}, \ldots, e_{n}$ denote the standard basis of $\mathbb{R}^{n}$, and let $v_{i}=L\left(e_{i}\right)$ for $i=$ $1, \ldots, n$. Let $\mathcal{J}$ denote the set of subsets $J \subset\{1, \ldots, n\}$ for which the vectors $v_{j}$, for $j \in J$, are linearly independent.

For each $J \in \mathcal{J}$, let $\mathbb{R}_{+}^{J}=\left\{s \in \mathbb{R}_{+}^{n} \mid s_{j}=0\right.$ for all $\left.j \notin J\right\}$, and let $C_{J}=L\left(\mathbb{R}_{+}^{J}\right)$. Also, for $\varepsilon>0$, let $B_{\varepsilon}$ denote the ball in $\mathbb{R}^{k}$ of radius $\varepsilon$ centered at the origin.

For every $J \in \mathcal{J}$, the map $L$ restricts to a homeomorphism from $\mathbb{R}_{+}^{J}$ to $C_{J}$. So $L\left(\Lambda \cap \mathbb{R}_{+}^{J}\right)$ is open in $C_{J}$ and contains the origin. Let $\varepsilon_{J}$ be a positive number such that $L\left(\Lambda \cap \mathbb{R}_{+}^{J}\right)$ contains $C_{J} \cap B_{\varepsilon_{J}}$. By Lemma 8.3, the union of the sets $C_{J}$, for $J \in \mathcal{J}$, is all of $C$. Let $\varepsilon=\min _{J \in \mathcal{J}} \varepsilon_{J}$. Then $L(\Lambda)$ contains $C \cap B_{\varepsilon}$.

8.5 Lemma Let $L: \mathbb{R}^{n} \rightarrow \mathbb{R}^{k}$ be a linear map, let $0 \leq r \leq n$, let $S_{r}^{n}=\mathbb{R}_{+}^{r} \times \mathbb{R}^{n-r}$ be a sector, and let $C=L\left(S_{r}^{n}\right)$ be its image. Let $\mathcal{O}$ be a neighborhood of the origin in $S_{r}^{n}$. Then $L(\mathcal{O})$ is a relative neighborhood of the origin in $C$. 
Proof For every $o=\left(o_{1}, \ldots, o_{n}\right) \in\{1\}^{r} \times\{-1,1\}^{n-r}$, let $F_{o}: \mathbb{R}^{n} \rightarrow \mathbb{R}^{n}$ be the map

$$
F_{o}\left(s_{1}, \ldots, s_{n}\right)=\left(o_{1} s_{1}, \ldots, o_{n} s_{n}\right) .
$$

The cone $C$ is the union, over all $o \in\{1\}^{r} \times\{-1,1\}^{n-r}$, of the sets

$$
C_{o}:=L\left(F_{o}\left(\mathbb{R}_{+}^{n}\right)\right) \text {. }
$$

Let $\rho_{o}$ be a positive number such that $F_{o}\left(\rho_{o} \Lambda\right)$ is contained in $\mathcal{O}$. It exists because $F_{o} \mid \mathbb{R}_{+}^{n}$ is continuous and carries 0 into the open set $\mathcal{O}$, and because every neighborhood of 0 in $\mathbb{R}_{+}^{n}$ contains a set of the form $\rho \Lambda$ for some $\rho>0$.

By Lemma 8.4, applied to the linear map $x \mapsto L\left(F_{o}\left(\rho_{0} x\right)\right)$, there exists $\varepsilon_{o}>0$ such that $L\left(F_{o}\left(\rho_{o} \Lambda\right)\right)$ contains $B_{\varepsilon_{o}} \cap C_{o}$. Let $\varepsilon=\min _{o \in O} \varepsilon_{o}$. Then $L(\mathcal{O})$ contains $B_{\varepsilon} \cap C$.

8.6 Lemma Let $L: \mathbb{R}^{n} \rightarrow \mathbb{R}^{k}$ be a linear map, and let $C=L\left(\mathbb{R}_{+}^{n}\right)$. Then the map

$$
\left.L\right|_{\mathbb{R}_{+}^{n}}: \mathbb{R}_{+}^{n} \rightarrow C
$$

is open.

Proof Let $x \in \mathbb{R}_{+}^{n}$. Without loss of generality we may assume that $x_{j}=0$ for all $1 \leq j \leq r$ and $x_{j}>0$ for all $r+1 \leq j \leq n$.

Let $\mathcal{O}$ be a sufficiently small neighborhood of the origin in the sector $S_{r}^{n}$ so that the translation $x+\mathcal{O}$ is contained in $\mathbb{R}_{+}^{n}$. Then $x+\mathcal{O}$ is a neighborhood of $x$ in $\mathbb{R}_{+}^{n}$.

By Lemma 8.5, $L(\mathcal{O})$ is a neighborhood of the origin in $L\left(S_{r}^{n}\right)$. This implies that $L(x+\mathcal{O})$ is a neighborhood of $L(x)$ in $C$. Indeed, let $\varepsilon>0$ be such that $L(\mathcal{O})$ contains $B_{\varepsilon} \cap L\left(S_{r}^{n}\right)$. Then $L(x+\mathcal{O})$ contains the $\varepsilon$-neighborhood of $L(x)$ in $C$. This follows from the fact that, for every $y \in C$, the difference $y-L(x)$ is in $L\left(S_{r}^{n}\right)$. This shows that the map $\left.L\right|_{\mathbb{R}_{+}^{n}}: \mathbb{R}_{+}^{n} \rightarrow C$ is open.

The closure of $\Lambda$ in $\mathbb{R}^{n}$ is the simplex

$$
\bar{\Lambda}=\left\{s \in \mathbb{R}_{+}^{n} \mid s_{1}+\cdots+s_{n} \leq 1\right\} .
$$

8.7 Lemma Let $L: \mathbb{R}^{n} \rightarrow \mathbb{R}^{k}$ be a linear map, $A=L(\Lambda), \bar{A}=L(\bar{\Lambda})$, and $A^{\mathrm{ext}}=$ $\bar{A} \backslash A$. Then:

(1) For every $\beta \in A \backslash\{0\}$, the intersection $\mathbb{R}_{+} \beta \cap A^{\text {ext }}$ contains exactly one point; call it $\beta^{\mathrm{ext}}$.

(2) $\beta \mapsto \beta^{\text {ext }}$ defines a continuous map from $A \backslash\{0\}$ to $A^{\text {ext }}$.

8.8 Remark The notation "ext" stands for "extremal". 
Proof of Lemma 8.7 Fix $\beta \in A \backslash\{0\}$. Because the subset $\bar{A}$ of $\mathbb{R}^{k}$ is closed, bounded, and contains $\beta$, the set

$$
\left\{t \in(0,1] \mid \frac{1}{t} \beta \in \bar{A}\right\}
$$

has a positive minimum; call it $t_{\beta}$. Note that $\left(1 / t_{\beta}\right) \beta$ is in $\mathbb{R}_{+} \cdot \beta \cap \bar{A}$. So, for (1), it is enough to show that $\left(1 / t_{\beta}\right) \beta$ is not in $A$. Equivalently, it is enough to show that if $(1 / t) \beta$ is in $A$, then $t$ is not minimal in (8.9). Suppose now that $(1 / t) \beta$ is in $A$. Write it as $\sum s_{j} v_{j}$ where the coefficients $s_{j}$ are nonnegative and where $\sum s_{j}<1$. Then $\left(\sum s_{j}\right) t$ is strictly smaller than $t$ and $\left(1 /\left(\sum s_{j}\right) t\right) \beta$ is in $\bar{A}$, so $t$ is not minimal in (8.9). This proves (1).

To prove (2), suppose that $\beta_{n}$ is a sequence of elements of $A \backslash\{0\}$, let $\beta_{n}^{\text {ext }}$ be their images in $A^{\text {ext }}$, suppose that the sequence $\beta_{n}$ converges to an element $\beta_{\infty}$ of $A \backslash\{0\}$, and suppose that the sequence $\beta_{n}^{\text {ext }}$ converges to an element $\beta^{\prime}$ of $\mathbb{R}^{k}$.

Lemma 8.6 implies that $A$ is open in $L\left(\mathbb{R}_{+}^{n}\right)$, and hence in $\bar{A}$. This, in turn, implies that $A^{\text {ext }}$ is closed in $\bar{A}$, and hence in $\mathbb{R}^{k}$. Thus, $\beta^{\prime}$ must be in $A^{\text {ext }}$. In particular, $\beta^{\prime}$ is nonzero.

Because $\beta_{n}^{\text {ext }} \in \mathbb{R}_{+} \beta_{n}$ and $\beta_{n} \rightarrow \beta_{\infty}$ as $n \rightarrow \infty$, we have $\beta_{n}^{\text {ext }} /\left\|\beta_{n}^{\text {ext }}\right\| \rightarrow \beta_{\infty} /\left\|\beta_{\infty}\right\|$ as $n \rightarrow \infty$. Because $\beta_{n}^{\text {ext }} \rightarrow \beta^{\prime}$ as $n \rightarrow \infty$, we have $\beta_{n}^{\text {ext }} /\left\|\beta_{n}^{\text {ext }}\right\| \rightarrow \beta^{\prime} /\left\|\beta^{\prime}\right\|$ as $n \rightarrow \infty$. By uniqueness of the limit, we deduce that $\beta^{\prime} \in \mathbb{R}_{+} \beta_{\infty}$. Because $A^{\text {ext }}$ intersects every ray in at most one point, we must have $\beta^{\prime}=\beta_{\infty}^{\text {ext }}$.

Now suppose that $\beta_{n}$ is any sequence of elements of $A \backslash\{0\}$ that converges to an element $\beta_{\infty}$ of $A \backslash\{0\}$. The above argument implies that $\beta_{\infty}^{\text {ext }}$ is the limit of every converging subsequence of $\beta_{n}^{\text {ext }}$. Because $\beta_{n}^{\text {ext }}$ are in $A^{\text {ext }}$ and the set $A^{\text {ext }}$ is compact, this implies that the sequence $\beta_{n}^{\text {ext }}$ converges to $\beta_{\infty}^{\text {ext }}$.

8.10 Lemma Let $L: \mathbb{R}^{n} \rightarrow \mathbb{R}^{k}$ be a linear map. Then $\left.L\right|_{\Lambda}$ has a continuous section. That is, there exists a continuous map $\sigma: A \rightarrow \Lambda$, where $A=L(\Lambda)$, such that $L \circ \sigma=$ $\operatorname{id}_{A}$.

Proof First, we show that the map

$$
\left.L\right|_{\bar{\Lambda}}: \bar{\Lambda} \rightarrow \bar{A}
$$

has a continuous section:

$$
\bar{\sigma}: \bar{A} \rightarrow \bar{\Lambda}, \quad L \circ \bar{\sigma}=\mathrm{id}_{\bar{A}} .
$$

We define $\bar{\sigma}$ recursively on the faces of $\bar{A}$. First, we define it, arbitrarily, on vertices. Now, suppose that $Q$ is a face of $\bar{A}$ and that we already defined $\bar{\sigma}$ on the relative 
boundary $\partial Q$ of $Q$. We define $\bar{\sigma}$ arbitrarily at a point $q$ in the relative interior of $Q$, and we extend it in an affine manner on segments connecting $q$ to $\partial Q$.

Next, we restrict this section to the closed subset $A^{\text {ext }}$ to obtain a continuous map

$$
\sigma^{\mathrm{ext}}: A^{\mathrm{ext}} \rightarrow \bar{\Lambda}
$$

such that $L \circ \sigma^{\mathrm{ext}}=\mathrm{id}_{A^{\mathrm{ext}}}$.

Finally, suppose that $\beta \in A \backslash\{0\}$ and let $\beta^{\text {ext }}$ be its image in $A^{\text {ext }}$. Then $\beta=t_{\beta} \beta^{\text {ext }}$. A priori $t_{\beta} \in(0,1]$, but, because $\beta$ itself is not in $A^{\text {ext }}$, the number $t_{\beta}$ is strictly less than one. We then define $\sigma(\beta)=t_{\beta} \sigma^{\text {ext }}\left(\beta^{\text {ext }}\right)$.

Because $\sigma(\beta)$ is the product of an element of $\bar{\Lambda}$ with a positive number that is strictly less than one, it is in $\Lambda$. So $\sigma$ is a map from $A$ to $\Lambda$.

By Lemma 8.7, the map $\beta \mapsto \beta^{\text {ext }}$ is continuous; it follows that $\beta \mapsto t_{\beta}$ is also continuous. Thus, $\sigma: A \rightarrow \Lambda$ is continuous.

Finally, $L(\sigma(\beta))=L\left(t_{\beta} \sigma^{\text {ext }}\left(\beta^{\text {ext }}\right)\right)=t_{\beta} \beta^{\text {ext }}=\beta$. So $L \circ \sigma=\mathrm{id}_{A}$, and $\sigma$ is a section of $\left.L\right|_{\Lambda}: \Lambda \rightarrow A$, as required.

Proof of Proposition 8.1 By Lemma 8.6, the map $L{\mid \mathbb{R}_{+}^{n}}_{\Lambda^{\prime}}$ is open as a map to its image. Because $\Lambda$ is open in $\mathbb{R}_{+}^{n}$, this implies that the map $\left.L\right|_{\Lambda}$ is also open as a map to its image.

Because $\Lambda$ is convex and $L$ is linear, the level sets of $\left.L\right|_{\Lambda}$ are path connected.

By Lemma 8.10, the map $\left.L\right|_{\Lambda}: \Lambda \rightarrow A$ has a continuous section. This together with the connectedness of the level sets implies the weak path lifting property.

8.11 Corollary Let $v_{1}, \ldots, v_{n}$ be vectors in $\mathbb{R}^{k}$. Let $B_{\varepsilon}$ be an open $\varepsilon$-ball about 0 in $\mathbb{R}^{\ell}$. Let

$$
\begin{aligned}
& \Lambda=\left\{s \in \mathbb{R}^{n} \mid s_{j} \geq 0 \text { for } j=1, \ldots, n, \text { and } \sum_{j=1}^{n} s_{j}<1\right\}, \\
& A=\left\{\sum_{j=1}^{n} s_{j} v_{j} \mid s_{j} \geq 0 \text { for } j=1, \ldots, n, \text { and } \sum_{j=1}^{n} s_{j}<1\right\},
\end{aligned}
$$

Then the map

$$
\varphi: \Lambda \times B_{\varepsilon} \rightarrow A \times B_{\varepsilon} \quad \text { given by } \quad\left(\left(s_{1}, \ldots, s_{n}\right), \eta\right) \mapsto\left(\sum_{j=1}^{n} s_{j} v_{j}, \eta\right)
$$

is open, has the weak lifting property, and its level sets are path connected. 
Proof These properties follow from the analogous properties of the map $s \mapsto \sum s_{j} v_{j}$ from $\Lambda$ to $A$, which, in turn, were established in Proposition 8.1.

\section{Contact momentum maps}

An exact symplectic manifold is a symplectic manifold $(Q, \omega)$ such that the symplectic form $\omega$ is exact: there exists a one-form $\alpha$ such that $\omega=d \alpha$. Let a torus $T$ act on an exact symplectic manifold $(Q, \omega)$ and, for every Lie algebra element $X \in \mathfrak{t}$, let $X_{Q}$ be the corresponding vector field on $Q$. Suppose that $\omega=d \alpha$ and that $\alpha$ is $T$-invariant. Then the map $\Phi: Q \rightarrow \mathfrak{t}^{*}$ given by

$$
\Phi^{X}(q)=\alpha\left(X_{Q}(q)\right)
$$

for all $X \in \mathfrak{t}$ and $q \in Q$ is a momentum map: $d \Phi^{X}=-\iota\left(X_{Q}\right) \omega$. An exact momentum map is a momentum map that has this form.

We recall from Section 1 that, if a torus $T$ acts on a manifold $M$ and preserves a contact one-form $\alpha$, the $\alpha$-momentum map is the map $\Psi_{\alpha}: M \rightarrow \mathfrak{t}^{*}$ defined by $\Psi_{\alpha}^{X}=\iota\left(X_{M}\right) \alpha$, and the contact momentum map is the map $\Psi: M \times \mathbb{R}_{>0} \rightarrow \mathrm{t}^{*}$ defined by $\Psi(x, t)=t \Psi_{\alpha}(x)$. The map $\Psi$ is an exact momentum map on the symplectization $\left(M \times \mathbb{R}_{>0}, d(t \alpha)\right)$, with the trivial extension of the $T$ action to $M \times \mathbb{R}_{>0}$, and the map $\Psi_{\alpha}$ is a momentum map for the closed (degenerate) two-form $d \alpha$ on $M$.

9.2 Lemma Let a torus $T$ act on an exact symplectic manifold $(Q, \omega)$ with exact momentum map $\Phi: Q \rightarrow \mathfrak{t}^{*}$. Let $q$ be a point of $Q$. Then every neighborhood of $q$ in $Q$ contains a smaller neighborhood $U$ such that:

(1) The map $\left.\Phi\right|_{U}: U \rightarrow \Phi(U)$ is open, has the weak path lifting property (cf Definition 7.9), and its level sets are path connected.

(2) There exists a convex polyhedral cone $C_{q}$ with vertex at the origin, such that the set $\Phi(U)$ is a relatively open subset of $C_{q}$.

Proof Let $H$ denote the stabilizer of $q$. Let $\mathfrak{h}$ denote its Lie algebra, $\mathfrak{h}^{*}$ the dual space, and $\mathfrak{h}^{0}$ the annihilator of $\mathfrak{h}$ in $\mathfrak{t}^{*}$. Fix an inner product on $\mathfrak{t}$ and use it to identify $\mathfrak{h}^{*}$ with a subspace of $\mathfrak{t}^{*}$. By the local normal form theorem for Hamiltonian torus actions, there exists an action of $H$ on $\mathbb{C}^{n}$, with weights $\eta_{1}, \ldots, \eta_{n} \in \mathfrak{h}^{*}$, there exists a $T$-invariant symplectic form on the model $Y:=T \times_{H} \mathbb{C}^{n} \times \mathfrak{h}^{0}$ (with the left $T$ action), and there exists an equivariant symplectomorphism from an invariant 
neighborhood of $q$ in $Q$ to an open subset of $Y$ that carries the point $q$ to $[1,0,0]$ and that carries the momentum map $\Phi$ to the map

$$
\Phi_{Y}([t, z, v])=\Phi(q)+\frac{\left|z_{1}\right|^{2}}{2} \eta_{1}+\cdots+\frac{\left|z_{n}\right|^{2}}{2} \eta_{n}+v .
$$

The image of $\Phi_{Y}$ is the translation of the cone

$$
C_{q}=\left\{s_{1} \eta_{1}+\cdots+s_{n} \eta_{n}+v \mid s_{j} \geq 0 \text { for all } j, \text { and } v \in \mathfrak{h}^{0}\right\}
$$

by the element $\Phi(q)$ of $\mathfrak{t}^{*}$. The cone $C_{q}$ is a convex polyhedral cone in $\mathfrak{t}^{*}$, with vertex at the origin, invariant under translations by elements of $\mathfrak{h}^{0}$. By the formula (9.1) for the exact momentum map, the element $\Phi(q)$ of $\mathfrak{t}^{*}$ belongs to the annihilator $\mathfrak{h}^{0}$ of $\mathfrak{h}$. It follows that the image of $\Phi_{Y}$ is equal to $C_{q}$.

We need to show that the restriction of $\Phi$ to arbitrarily small neighborhoods $U$ of $q$ in $Q$ satisfies (1) and (2). By the local normal form theorem, it is enough to show these properties for the restriction of $\Phi_{Y}$ to neighborhoods of $[1,0,0]$ in $Y$.

For $\varepsilon>0$, let $B_{\varepsilon}^{T}$ be the $\varepsilon$-neighborhood of the unit element in $T$ (with respect to some invariant metric); so $B_{\varepsilon}^{T} \cdot H$ is the $\varepsilon$-neighborhood of $H$ in $T$; let $B_{\varepsilon}^{\mathbb{C}^{n}}$ be the $\varepsilon$-ball about the origin in $\mathbb{C}^{n}$; and let $B_{\varepsilon}^{\mathfrak{h}^{0}}$ be the $\varepsilon$-ball about the origin in $\mathfrak{h}^{0}$. Let

$$
U_{\varepsilon}:=\left(B_{\varepsilon}^{T} \cdot H\right) \times_{H} B_{\varepsilon}^{\mathbb{C}^{n}} \times B_{\varepsilon}^{\mathfrak{h}^{0}} .
$$

Because every neighborhood of $[1,0,0]$ in $Y$ contains a set of the form $U_{\varepsilon}$ for some $\varepsilon>0$, it is enough to show that:

(1') The map $\left.\Phi_{Y}\right|_{U_{\varepsilon}}: U_{\varepsilon} \rightarrow \Phi_{Y}\left(U_{\varepsilon}\right)$ is open, has the weak path lifting property, and its level sets are path connected.

$\left(2^{\prime}\right)$ The set $\Phi_{Y}\left(U_{\varepsilon}\right)$ is a neighborhood of $\Phi(q)$ in the cone $C_{q}$.

The map $\left(z_{1}, \ldots, z_{n}\right) \mapsto\left(s_{1}, \ldots, s_{n}\right)$, where $\left|z_{j}\right|^{2}=\varepsilon^{2} s_{j}$, takes $B_{\varepsilon}^{\mathbb{C}^{n}}$ onto the set

$$
\Lambda:=\left\{s \in \mathbb{R}_{+}^{n} \mid s_{1}+\cdots+s_{n}<1\right\} .
$$

By $(9.3)$,

$$
\Phi_{Y}\left(U_{\varepsilon}\right)=\left\{\Phi(q)+\frac{\varepsilon^{2}}{2} \sum s_{j} \eta_{j}+v \mid\left(s_{1}, \ldots, s_{n}\right) \in \Lambda \text { and } v \in B_{\varepsilon}^{\mathfrak{h}^{0}}\right\} .
$$

The affine isomorphism

$$
(\beta, v) \mapsto \Phi(q)+\frac{\varepsilon^{2}}{2} \beta+v
$$


of $\mathfrak{h}^{*} \times \mathfrak{h}^{0}$ with $\mathfrak{t}^{*}$ carries the cone $\left\{\sum s_{j} \eta_{j} \mid s_{j} \geq 0\right.$ for all $\left.j\right\} \times \mathfrak{h}^{0}$ to the cone $C_{q}$ and the subset $A \times B_{\varepsilon}^{\mathfrak{h}^{0}}$, where

$$
A:=\left\{\sum s_{j} \eta_{j} \mid\left(s_{1}, \ldots, s_{n}\right) \in \Lambda\right\},
$$

to $\Phi_{Y}\left(U_{\varepsilon}\right)$. Because, by Lemma 8.4 , the set $A$ is open in the cone $\left\{\sum s_{j} \eta_{j} \mid\right.$ $s_{j} \geq 0$ for all $\left.j\right\}$, this gives $\left(2^{\prime}\right)$.

The map

given by

$$
B_{\varepsilon}^{T} \times \Lambda \times\left(S^{1}\right)^{n} \times B_{\varepsilon}^{\mathfrak{h}^{0}} \longrightarrow U_{\varepsilon}
$$

$$
\left(\lambda,\left(s_{1}, \ldots, s_{n}\right),\left(e^{i \theta_{1}}, \ldots, e^{i \theta_{n}}\right), v\right) \mapsto[\lambda, z, v] \quad \text { where } z_{j}=\varepsilon \sqrt{s_{j}} e^{i \theta_{j}}
$$

is continuous and onto. So, for $\left(1^{\prime}\right)$, it is enough to show that the composition of this map with $\Phi_{Y}$, as a map to $\Phi_{Y}\left(U_{\varepsilon}\right)$, is open, has the weak path lifting property, and its level sets are path connected.

This composition can be expressed as the composition of the map

$$
B_{\varepsilon}^{T} \times \Lambda \times\left(S^{1}\right)^{n} \times B_{\varepsilon}^{\mathfrak{h}^{0}} \stackrel{\text { projection }}{\longrightarrow} \Lambda \times B_{\varepsilon}^{\mathfrak{h}^{0}}
$$

with the map

$$
\Lambda \times B_{\varepsilon}^{\mathfrak{h}^{0}} \stackrel{(s, v) \mapsto\left(\sum s_{j} \eta_{j}, v\right)}{\longrightarrow} A \times B_{\varepsilon}^{\mathfrak{h}^{0}}
$$

and the map

$$
A \times B_{\varepsilon}^{\mathfrak{h}^{0}} \stackrel{(\beta, v) \mapsto \Phi(q)+\left(\varepsilon^{2} / 2\right) \beta+v}{\longrightarrow} \Phi_{Y}\left(U_{\varepsilon}\right) .
$$

So, for $\left(1^{\prime}\right)$, it is enough to check that each of the maps (9.4), (9.5) and (9.6) is continuous, open, onto, has the weak path lifting property, and its level sets are path connected. The map (9.4) is a fibration with path connected fibers, and the map (9.6) is a homeomorphism, so they both have the required properties. The required properties of the map (9.5) follow from Corollary 8.11.

9.7 Lemma Let a torus $T$ act on a contact manifold $(M, \xi=\operatorname{ker} \alpha)$ with $\alpha-$ momentum map $\Psi_{\alpha}: M \rightarrow \mathfrak{t}^{*}$. Then, for every point $x$ of $M$ with $\Psi_{\alpha}(x)=0$, there exists an open neighborhood $U_{x}$ of $x$ in $M$ and a convex polyhedral cone $C_{x}$ in $t^{*}$ with vertex at the origin such that:

- The image $\Psi_{\alpha}\left(U_{x}\right)$ is an open subset of $C_{x}$.

- The map $\left.\Psi_{\alpha}\right|_{U_{x}}: U_{x} \rightarrow \Psi_{\alpha}\left(U_{x}\right)$ is open, has the weak path lifting property, and its level sets are path connected. 
Proof Let $x$ be a point in $M$ with $\Psi_{\alpha}(x)=0$. Let $R_{\alpha}$ be the Reeb vector field of the contact form $\alpha$. Recall that it is defined by the conditions $\iota\left(R_{\alpha}\right) d \alpha=0$ and $\alpha\left(R_{\alpha}\right)=1$. The null space of $\left.d \alpha\right|_{T_{x} M}$ is $\mathbb{R} R_{\alpha}(x)$. Because $x$ is in the zero level set of the $\alpha$-momentum map, the tangent space to its orbit, $T_{x}(T \cdot x)$, is contained in the contact distribution, so $\mathbb{R} R_{\alpha}(x) \cap T_{x}(T \cdot x)=\{0\}$.

Let $H$ be the stabilizer of $x$; it acts linearly on $T_{x} M$. Let $W$ be an $H$-invariant subspace of $T_{x} M$ that is complementary to $\mathbb{R} R_{\alpha}(x) \oplus T_{x}(T \cdot x)$. Then we have an $H$-invariant decomposition

$$
T_{x} M=\mathbb{R} R_{\alpha}(x) \oplus T_{x}(T \cdot x) \oplus W,
$$

and $d \alpha$ is nondegenerate on $T_{x}(T \cdot x) \oplus W$.

Let $\psi$ be an $H$-equivariant diffeomorphism from a neighborhood of the origin in $T_{x} M$ to a neighborhood of $x$ in $M$ whose differential at $x$ is the identity map on $T_{x} M$.

Denote the Reeb trajectory of a point $q$ by $q^{(t)}$. Thus, $q^{(0)}=q$ and $\frac{d}{d t} q^{(t)}=R_{\alpha}\left(q^{(t)}\right)$. Then, for the interval $I=(-\varepsilon, \varepsilon)$ with sufficiently small $\varepsilon>0$ and for a sufficiently small neighborhood $D$ of the origin in $W$, the formula $(t,[a, u]) \mapsto(a \cdot \psi(u))^{(t)}$ defines a diffeomorphism from $I \times\left(T \times{ }_{H} D\right)$ to an open subset of $M$, and the image of $\{0\} \times\left(T \times{ }_{H} D\right)$ under this diffeomorphism is a submanifold of $M$ on which $d \alpha$ is nondegenerate.

We denote this submanifold by $Q$, the inclusion map by $i: Q \rightarrow M$, the symplectic form by $\omega_{Q}:=i^{*} d \alpha$, and the momentum map by $\Phi_{Q}:=i^{*} \Psi_{\alpha}$.

The map $f: I \times Q \rightarrow M$, given by $(t, q) \mapsto q^{(t)}$, is a $T$-equivariant diffeomorphism to an invariant open subset of $M$, and the pullback of $d \alpha$ through this diffeomorphism is equal to the pullback of $\omega_{Q}$ with respect to the projection map $I \times Q \rightarrow Q$. It follows that the pullback $f^{*} \Psi_{\alpha}$ must have the form $(t, q) \mapsto \Phi_{Q}(q)$. The properties of $\Psi_{\alpha}$ then follow from the corresponding properties of $\Phi_{Q}$, which are guaranteed by Lemma 9.2.

9.8 Remark For a contact manifold with compact group action, Loose [28, Theorem 3] gives a local normal form that describes the neighborhood of an orbit in the zero level set, up to equivariant contactomorphism. (Without a contact one-form, he defines a momentum map with values in $\mathfrak{g}^{*} \otimes L$, where $L$ is the line bundle $T M / \xi$ over $M$.) 
9.9 Lemma Let a torus $T$ act on a contact manifold $(M, \xi=\operatorname{ker} \alpha)$ with $\alpha-$ momentum map $\Psi_{\alpha}: M \rightarrow \mathfrak{t}^{*}$ and contact momentum map $\Psi: M \times \mathbb{R}_{>0} \rightarrow \mathfrak{t}^{*}$.

Choose a metric on $\mathfrak{t}^{*}$ and let $S\left(\mathfrak{t}^{*}\right)$ denote the unit sphere in $\mathrm{t}^{*}$. Define the map $\bar{\Psi}:\left(M \backslash \Psi_{\alpha}^{-1}(0)\right) \times \mathbb{R}_{>0} \rightarrow S\left(\mathfrak{t}^{*}\right)$ by $\bar{\Psi}=\Psi /\|\Psi\|$.

Then, for any $(x, \lambda) \in M \times \mathbb{R}_{>0}$ with $\Psi_{\alpha}(x) \neq 0$, every neighborhood of $(x, \lambda)$ in $M \times \mathbb{R}_{>0}$ contains a smaller neighborhood $U$ such that:

- The map $\left.\Psi\right|_{U}$ is convex.

- The image $\Psi(U)$ is a relatively open subset in a convex polyhedral cone with vertex at the origin.

- The map $\left.\bar{\Psi}\right|_{U}$ is (defined and) open as a map to its image.

Proof Let $x \in M \backslash \Psi_{\alpha}^{-1}(0)$. Because $\Psi$ is an exact momentum map, by Lemma 9.2, every neighborhood of $(x, \lambda)$ in $M \times \mathbb{R}_{>0}$ contains a smaller neighborhood $U^{\prime}$ such that the map $\left.\Psi\right|_{U^{\prime}}: U^{\prime} \rightarrow \Psi\left(U^{\prime}\right)$ is open, has the weak path lifting property, its level sets are path connected, and its image has the form $\mathcal{O} \cap C_{(x, \lambda)}$ where $\mathcal{O}$ is an open neighborhood of $\Psi(x, \lambda)$ and $C_{(x, \lambda)}$ is a convex polyhedral cone with vertex at the origin. We may assume $\mathcal{O}$ does not contain the origin. Let $B$ be a convex open neighborhood of $\Psi(x, \lambda)$ that is contained in $\mathcal{O}$, and let $U$ be the intersection of $U^{\prime}$ with the preimage of $B$. Then $\left.\Psi\right|_{U}: U \rightarrow \Psi(U)$ still has the weak path lifting property and its level sets are path connected, but, additionally, its image, being equal to $B \cap C_{(x, \lambda)}$, is convex. These properties imply that $\left.\Psi\right|_{U}$ is convex. Since $\left.\Psi\right|_{U}: U \rightarrow \Psi(U)$ is still open and the image $\Psi(U)=B \cap C_{(x, \lambda)}$ is open in $C_{(x, \lambda)}$, the map $\left.\Psi\right|_{U}: U \rightarrow C_{(x, \lambda)}$ is a continuous nonvanishing open map. By Lemma 6.7, the map $\left.\bar{\Psi}\right|_{U}: U \rightarrow C_{(x, \lambda)} \cap S\left(\mathfrak{t}^{*}\right)$ is open. This implies that $\left.\bar{\Psi}\right|_{U}$ is open as a map to its image.

9.10 Lemma Let $C$ be a closed convex cone in $\mathbb{R}^{n}$ with vertex at the origin. Suppose that for every $w$ in $C \backslash\{0\}$ there exists a neighborhood $U_{w}$ in $\mathbb{R}^{n}$ and a convex polyhedral cone $C_{w}$ with vertex at the origin such that $U_{w} \cap C=U_{w} \cap C_{w}$. Then $C$ is a convex polyhedral cone with vertex at the origin.

Proof After possibly shrinking the neighborhoods $U_{w}$, we may assume that these neighborhoods are convex. We may also assume that $\mathbb{R}_{>0} \cdot U_{w}=U_{w}$. Otherwise, we replace $U_{w}$ by $\mathbb{R}_{>0} \cdot U_{w}$; it remains an open set that satisfies $U_{w} \cap C=U_{w} \cap C_{w}$.

Recall that a convex polyhedral cone with vertex at the origin is a finite intersection of closed half-spaces whose boundaries contain the origin. 
For $w \in C$, let $H_{w}^{j}$, for $1 \leq j \leq N_{w}$, be closed half-spaces whose boundaries contain the origin and such that $C_{w}=H_{w}^{1} \cap \cdots \cap H_{w}^{N_{w}}$. (It is possible that $N_{w}=0$ and $C_{w}=\mathbb{R}^{n}$.) Let $\partial H_{w}^{j}$ denote the boundary of $H_{w}^{j}$. We may assume that $U_{w} \cap C \cap \partial H_{w}^{j} \neq \varnothing$ for every $1 \leq j \leq N_{w}$. Otherwise, we replace the polyhedral cone $C_{w}$ by the intersection of those $H_{w}^{j}$ that do satisfy $U_{w} \cap C \cap \partial H_{w}^{j} \neq \varnothing$; this intersection is a (possibly larger) cone that still satisfies $U_{w} \cap C=U_{w} \cap C_{w}$.

Since $C \cap S^{n-1}$ is compact, we may choose a finite set of points $W=\left\{w_{1}, \ldots, w_{N}\right\} \subset$ $C \cap S^{n-1}$ such that $U_{w_{1}} \cup \cdots \cup U_{w_{N}}$ contains $C \cap S^{n-1}$. We claim that

$$
C=\bigcap_{\substack{w \in W \\ 1 \leq j \leq N_{w}}} H_{w}^{j} .
$$

Fix $w \in W$ and $1 \leq j \leq N_{w}$. Let $c$ be a point in $C$. Let $c^{\prime}$ be a point in $U_{w} \cap C \cap \partial H_{w}^{j}$. Because $C$ is convex, the segment $\left[c, c^{\prime}\right]$ is contained in $C$. Because $U_{w}$ is open, interior points of the segment that are sufficiently close to $c^{\prime}$ are in $U_{w}$. Let $c^{\prime \prime}$ be such a point. Because $c^{\prime \prime}$ is in $U_{w} \cap C$, it is in $H_{w}^{j}$. Finally, because $c^{\prime}$ is on the boundary of the half-space and $c^{\prime \prime}$ is in the half-space, $c$ is also in the half-space. Thus, $C \subset H_{w}^{j}$.

Denote the right hand side of (9.11) by $C_{\mathrm{RHS}}$. We have shown that $C \subset C_{\mathrm{RHS}}$. Because $C$ is closed in $\mathbb{R}^{n}$, it is closed in $C_{\mathrm{RHS}}$. Because $C$ is the union of the sets $U_{w} \cap C$ for $w \in W$, and because $U_{w} \cap C$ is open in $C_{w}$, hence in $C_{\mathrm{RHS}}$, we deduce that $C$ is open in $C_{\mathrm{RHS}}$. Because $C_{\mathrm{RHS}}$ is convex, hence connected, and $C$ is a nonempty subset that is both closed and open, $C$ is equal to $C_{\mathrm{RHS}}$.

The "convexity package" for contact momentum maps is given in parts (2), (3), (4) and (6) of the following theorem.

9.12 Theorem Let a torus $T$ act on a cooriented compact connected contact manifold $M$ with contact momentum map $\Psi: M \times \mathbb{R}_{>0} \rightarrow \mathfrak{t}^{*}$. Assume that the action is effective and the torus has dimension greater than 2 . Then:

(1) Let $y_{0}$ and $y_{1}$ be any two points in $M \times \mathbb{R}_{>0}$.

- If the action is transverse $(0 \notin$ image $\Psi)$, assume that the origin is not contained in the segment $\left[\Psi\left(y_{0}\right), \Psi\left(y_{1}\right)\right]$.

- If the action is not transverse $(0 \in$ image $\Psi)$, assume that $\Psi\left(y_{0}\right)$ and $\Psi\left(y_{1}\right)$ are not both zero.

Then there exists a path $\gamma:[0,1] \rightarrow M \times \mathbb{R}_{>0}$ such that $\gamma(0)=y_{0}$ and $\gamma(1)=y_{1}$ and such that $\Psi \circ \gamma:[0,1] \rightarrow \mathfrak{t}^{*}$ is a weakly monotone parametrization of the (possibly degenerate) segment $\left[\Psi\left(y_{0}\right), \Psi\left(y_{1}\right)\right]$.

(2) The momentum map $\Psi$ is open as a map to its image. 
Consequently:

(3) The momentum cone $C(\Psi)$ is convex.

(4) The nonzero level sets, $\Psi^{-1}(\mu)$, for $\mu \neq 0$, are connected.

(5) Let $A$ be a convex subset of $\mathfrak{t}^{*}$.

- If the action is transverse, suppose that $0 \notin A$.

- If the action is not transverse, suppose that $A \neq\{0\}$.

Then the preimage $\Psi^{-1}(A)$ is connected.

Moreover:

(6) The momentum cone $C(\Psi)$ is a convex polyhedral cone.

Proof Parts (3), (4) and (5) of the theorem follow easily from Part (1). We proceed to prove Parts (1), (2) and (6).

Write the contact momentum map $\Psi: M \times \mathbb{R}_{>0} \rightarrow \mathfrak{t}^{*}$ as $\Psi(x, t)=t \Psi_{\alpha}(x)$ where $\alpha$ is an invariant contact one-form and $\Psi_{\alpha}^{X}=\alpha\left(X_{M}\right)$ is the $\alpha$-momentum map. Choose a metric on $\mathfrak{t}^{*}$, denote the unit sphere by $S\left(\mathfrak{t}^{*}\right)$, and let

$$
\bar{\Psi}:=\Psi /\|\Psi\|:\left(M \backslash \Psi_{\alpha}^{-1}(0)\right) \times \mathbb{R}_{>0} \rightarrow S\left(\mathfrak{t}^{*}\right) .
$$

Because the $T$ action is effective on $M$, it is effective on $M \times \mathbb{R}_{>0}$. Because $M$ is connected, so is $M \times \mathbb{R}_{>0}$. Because $T$ is compact and abelian, by the principal orbit type theorem, there exists an invariant open dense subset of $M \times \mathbb{R}_{>0}$ on which the action is free. Wherever the action is free, the momentum map $\Psi$ is a submersion. Hence, the $\Psi$-image of any open subset of $M \times \mathbb{R}_{>0}$ is not contained in a proper subspace of $\mathrm{t}^{*}$. Because $\operatorname{dim} T>2$, and because $\Psi(x, t)=t \Psi_{\alpha}(x)$, this implies that the $\Psi_{\alpha}$-image of any open subset of $M$ is not contained in a two dimensional subspace of $t^{*}$.

Suppose that the action is transverse $\left(0 \notin \Psi_{\alpha}(M)\right)$.

Let $(x, \lambda)$ be a point in $M \times \mathbb{R}_{>0}$. By Lemma 9.9, there exists a neighborhood $U$ such that the map $\left.\Psi\right|_{U}$ is convex, $\Psi(U)$ is a relatively open subset in a convex polyhedral cone $C_{(x, \lambda)}$, and the map $\left.\bar{\Psi}\right|_{U}$ is open as a map to its image. Parts (1) and (2) of the theorem follow from Proposition 6.8, applied to the space $M$ and the map $\Psi_{\alpha}$.

Because $M$ is compact and $0 \notin$ image $\Psi_{\alpha}$, the momentum map $\Psi$ is proper as a map to $\mathfrak{t}^{*} \backslash\{0\}$, so its image is closed in $\mathfrak{t}^{*} \backslash\{0\}$. Part (6) of the theorem then follows from Lemma 9.10, applied to the momentum cone $C(\Psi)=\{0\} \cup$ image $\Psi$.

Now suppose that the action is not transverse $\left(0 \in \operatorname{image} \Psi_{\alpha}\right)$. 
- Let $x$ be a point of $M$ with $\Psi_{\alpha}(x)=0$. By Lemma 9.7, there exists a neighborhood $U_{x}$ of $x$ in $M$ and a convex polyhedral cone $C_{x}$ in $\mathrm{t}^{*}$ with vertex at the origin such that:

- The image $\Psi_{\alpha}\left(U_{x}\right)$ is an open subset of $C_{x}$.

- The map $\left.\Psi_{\alpha}\right|_{U_{x}}: U_{x} \rightarrow \Psi_{\alpha}\left(U_{x}\right)$ is open, has the weak path lifting property, and its level sets are path connected.

Because $\Psi_{\alpha}\left(U_{x}\right)$ is not contained in a two dimensional subspace of $t^{*}$ but is contained in $C_{x}$ :

- The cone $C_{x}$ is not contained in a two dimensional subspace of $\mathfrak{t}^{*}$.

- Let $(x, \lambda)$ be a point of $M \times \mathbb{R}_{>0}$ with $\Psi_{\alpha}(x) \neq 0$. By Lemma 9.9, every neighborhood of $(x, \lambda)$ in $M \times \mathbb{R}_{>0}$ contains a smaller neighborhood $U$ such that the map $\left.\Psi\right|_{U}$ is convex and the map $\left.\bar{\Psi}\right|_{U}$ is (defined and) open as a map to its image.

Parts (1), (2) and (6) of the theorem then follow from Proposition 7.11.

\section{Examples}

In the examples further below, we will need to know that a contact manifold is determined by its symplectization together with the $\mathbb{R}_{>0}$ action on the symplectization. We will also need to use the "contact cutting" construction. These are summarized in the following remark.

10.1 Remark Let $M$ be a $2 n+1$ dimensional manifold, $\pi: Q \rightarrow M$ a principal $\mathbb{R}_{>0}$ bundle, and $\omega$ a symplectic form on the total space $Q$ that is homogeneous of degree one with respect to the principal $\mathbb{R}_{>0}$ action. This structure is called a symplectic cone [18].

Let $\tilde{\alpha}=\iota_{v} \omega$, where $v$ is the vector field that generates the principal $\mathbb{R}_{>0}$ action. Then there exists a unique contact distribution $\xi$ on $M$ and a unique diffeomorphism from the symplectization $\xi_{+}^{0}$ onto $Q$ that respects the projection maps to $M$ and such that the pullback of $\widetilde{\alpha}$ is the tautological one-form on the subset $\xi_{+}^{0}$ of $T^{*} M$.

A torus $T$ action on $Q$ that commutes with the $\mathbb{R}_{>0}$ action and preserves $\omega$ descends to an action on the contact manifold $(M, \xi)$. The pullback to $Q$ of the contact momentum map is the map $\Psi: Q \rightarrow \mathfrak{t}^{*}$ given by $\Psi^{X}=\iota_{X_{Q}} \widetilde{\alpha}$, where $X_{Q}$, for $X \in \mathfrak{t}$, are the vector fields on $Q$ that generate the action.

Let $i: S^{1} \hookrightarrow T$ be a subcircle and $i^{*}: \mathfrak{t}^{*} \rightarrow \mathbb{R}$ the projection on the dual of the Lie algebra. Performing on $Q$ the symplectic cutting construction with respect to this 
circle action yields the symplectization of the contact cut of $M$; $\operatorname{cf}[14$, Theorem 6] and [23, Theorem 2.10]. Its momentum map image is the intersection of image $\Psi$ with the closed half-space $\left\{i^{*} \geq 0\right\}$ of $\mathfrak{t}^{*}$.

10.2 Example Consider $\mathbb{R}^{2 n} \backslash\{0\}$ as a principal $\mathbb{R}_{>0}$ bundle over $S^{2 n-1}$, where $t \in \mathbb{R}_{>0}$ acts by $x \mapsto \sqrt{t} x$ and where the map to $S^{2 n-1}$ is $x \mapsto x /\|x\|$, and with the standard symplectic structure. This is the symplectization of the standard contact structure on $S^{2 n-1}$ (cf Remark 10.1). The standard (Hopf) circle action has momentum map $x \mapsto\|x\|^{2} / 2$ with image $(0, \infty)$. The opposite circle action has momentum map with image $(-\infty, 0)$.

10.3 Example Consider the torus $T^{k}=\left(S^{1}\right)^{k}$; identify its cotangent bundle with $T^{k} \times \mathbb{R}^{k}$. Let $Q$ be the complement of the zero section: $Q=T^{k} \times\left(\mathbb{R}^{k} \backslash\{0\}\right)$. The subtorus $T^{k-1} \times\{1\}$ acts on $Q$ with momentum map $\Psi:(a, x) \mapsto \pi(x)$ where $\pi: \mathbb{R}^{k} \rightarrow \mathbb{R}^{k-1}$ is the projection to the first $(k-1)$ coordinates. The level sets of this momentum map are

$$
\Psi^{-1}(\beta)= \begin{cases}T^{k} \times\{\beta\} \times \mathbb{R} & \text { if } \beta \neq 0 \text { in } \mathbb{R}^{k-1}, \\ T^{k} \times\{0\} \times(\mathbb{R} \backslash\{0\}) & \text { if } \beta=0 \text { in } \mathbb{R}^{k-1} .\end{cases}
$$

In particular, the zero level set is not connected. This is an example of a contact momentum map: $Q$ is the symplectization of the unit sphere bundle in the cotangent bundle; cf Remark 10.1.

10.4 Example Begin with the symplectic manifold $Q$ of Example 10.3. By performing the symplectic cutting construction with respect to the circle $\{1\}^{k-2} \times S^{1} \times\{1\}$, we obtain a new symplectic manifold, $Q_{\text {cut }}$, still with a $(k-1)$ dimensional torus action and a momentum map $\Psi_{\text {cut }}: Q_{\text {cut }} \rightarrow \mathbb{R}^{k-1}$, but its momentum map image is now the closed upper half-space in $\mathbb{R}^{k-1}$ and not all of $\mathbb{R}^{k-1}$. This is the contact momentum map for the contact manifold obtained from the unit sphere bundle in the cotangent bundle by "contact cutting"; cf Remark 10.1. The zero level set of the momentum map is still disconnected. (Contrast with Remark 1.4.)

10.5 Example (Circle actions) Suppose that $\operatorname{dim} T=1$ and the contact manifold $M$ is connected. If $T$ acts effectively, the momentum map image must be one of the following sets: $(-\infty, 0),(-\infty, 0],(0, \infty),[0, \infty)$, or all of $\mathbb{R}$. As seen in Example 10.2, and in Examples 10.3 and 10.4 with $k=2$, all these sets occur as images, and the zero level set need not be connected. 
10.6 Example $(\operatorname{dim} T=2)$ For a two dimensional torus, the momentum cone need not be convex, and the level sets of the momentum map need not be connected. To see this, we begin with the noncompact manifold $\mathbb{R} \times\left(S^{1}\right)^{2}$, with $\left(S^{1}\right)^{2}$ acting by rotations of the second component, and with the contact one-form $\alpha=\cos t d \theta_{1}+\sin t d \theta_{2}$ and the $\alpha$-momentum map $\left(t, e^{i \theta_{1}}, e^{i \theta_{2}}\right) \mapsto(\cos t, \sin t)$. For every positive integer $n$, this descends to a contact one-form and torus action on the compact manifold $M_{n}=$ $\mathbb{R} /(2 \pi n \mathbb{Z}) \times\left(S^{1}\right)^{2}$. The image of the contact momentum map $M_{n} \times \mathbb{R}_{>0} \rightarrow \mathbb{R}^{2}$ is $\mathbb{R}^{2} \backslash\{0\}$, and every nonempty level set has $n$ connected components. Alternatively, for every interval $[a, b] \subset \mathbb{R}$ such that $\tan (a)$ and $\tan (b)$ are rational, contact cutting in neighborhoods of $\{a\} \times\left(S^{1}\right)^{2}$ and $\{b\} \times\left(S^{1}\right)^{2}$ produces a contact one-form and torus action on the lens space $M_{[a, b]}$, obtained from the manifold with boundary $[a, b] \times\left(S^{1}\right)^{2}$ by collapsing circles in the two components of the boundary by two circle subgroups. The image of the contact momentum map is $\{(r \cos t, r \sin t) \mid a \leq t \leq b$ and $r>0\}$. If $\pi<b-a<2 \pi$, the momentum cone is not convex. If $b-a \geq 2 \pi$, the contact momentum map is not open as a map to its image. These examples are due to Lerman [23]. Also see Example 6.11. We summarize this in Table 1.

\begin{tabular}{|l|c|c|c|}
\hline & convexity (C1) & connectedness (C2) & openness (C3) \\
\hline$M_{n}, n=1$ & $\checkmark$ & $\checkmark$ & $\checkmark$ \\
$M_{n}, n \geq 2$ & $\checkmark$ & $\times$ & $\checkmark$ \\
\hline$M_{[a, b]}, 0<b-a \leq \pi$ & $\checkmark$ & $\checkmark$ & $\checkmark$ \\
$M_{[a, b]}, \pi<b-a<2 \pi$ & $\times$ & $\checkmark$ & $\checkmark$ \\
$M_{[a, b]}, b-a \geq 2 \pi$ & $\checkmark$ & $\times$ & $\times$ \\
\hline
\end{tabular}

Table 1

We currently do not know whether the contact momentum map for a nontransverse $T$ action on a compact connected contact manifold can have disconnected nonzero level sets when $\operatorname{dim} T=2$. But it can have disconnected nonzero level sets when $\operatorname{dim} T=1$ :

10.7 Example (Nonzero level sets and openness for circle actions) For the manifolds of Example 10.6, restrict the torus action to an action of the circle $S^{1} \times\{1\}$; the momentum map gets composed with the projection map $(x, y) \mapsto x$. For the manifold $M_{n}$, the contact momentum map is open and its image is $\mathbb{R}$; the number of connected components of $\Psi^{-1}(x)$ is $n$ if $x \neq 0$ and $2 n$ if $x=0$. For the manifold $M_{[a, b]}$, where $b=b^{\prime}+2 \pi k$ with $k$ a nonnegative integer, the numbers of connected components of $\Psi^{-1}(x)$ and the images of $\Psi$ when $k=0$ are given in Table 2. 


\begin{tabular}{|c|c|c|c|c|c|}
\hline & & $x<0$ & $x=0$ & $x>0$ & $\begin{array}{c}\text { image } \Psi \\
\text { when } k=0\end{array}$ \\
\hline$a=-\pi / 2$ & $-\pi / 2<b^{\prime}<\pi / 2$ & $\mathrm{k}$ & $1+2 \mathrm{k}$ & $1+\mathrm{k}$ & {$[0, \infty)$} \\
\hline$a=-\pi / 2$ & $b^{\prime}=\pi / 2$ & $\mathrm{k}$ & $2+2 \mathrm{k}$ & $1+\mathrm{k}$ & {$[0, \infty)$} \\
\hline$a=-\pi / 2$ & $\pi / 2<b^{\prime}<3 \pi / 2$ & $1+\mathrm{k}$ & $2+2 \mathrm{k}$ & $1+\mathrm{k}$ & $\mathbb{R}$ \\
\hline$a=-\pi / 2$ & $b^{\prime}=3 \pi / 2$ & $1+\mathrm{k}$ & $3+2 \mathrm{k}$ & $1+\mathrm{k}$ & $\mathbb{R}$ \\
\hline$-\pi / 2<a<\pi / 2$ & $a<b^{\prime}<\pi / 2$ & $\mathrm{k}$ & $2 \mathrm{k}$ & $1+\mathrm{k}$ & $(0, \infty)$ \\
\hline$-\pi / 2<a<\pi / 2$ & $b^{\prime}=\pi / 2$ & $\mathrm{k}$ & $1+2 \mathrm{k}$ & $1+\mathrm{k}$ & {$[0, \infty)$} \\
\hline$-\pi / 2<a<\pi / 2$ & $\pi / 2<b^{\prime}<3 \pi / 2$ & $1+\mathrm{k}$ & $1+2 \mathrm{k}$ & $1+\mathrm{k}$ & $\mathbb{R}$ \\
\hline$-\pi / 2<a<\pi / 2$ & $b^{\prime}=3 \pi / 2$ & $1+\mathrm{k}$ & $2+2 \mathrm{k}$ & $1+\mathrm{k}$ & $\mathbb{R}$ \\
\hline$-\pi / 2<a<\pi / 2$ & $3 \pi / 2<b^{\prime} \leq a+2 \pi$ & $1+\mathrm{k}$ & $2+2 \mathrm{k}$ & $2+\mathrm{k}$ & $\mathbb{R}$ \\
\hline
\end{tabular}

Table 2

If $k \geq 1$, then image $\Psi=\mathbb{R}$. When we replace $[a, b]$ by $[a+\pi n, b+\pi n]$, if $n$ is even, we get the same values, and if $n$ is odd, the number of connected components for $x<0$ is switched with the number for $x>0$ and image $\Psi$ transforms by $x \mapsto-x$. When the image of the momentum map is $\mathbb{R}$ and $a$ or $b$ is equal to $\pi / 2$ modulo $\pi \mathbb{Z}$, the contact momentum map $\Psi$ is not open as a map to its image; in all other cases, the contact momentum map is open as a map to its image. We summarize this in Tables 3 and 4 . (The convexity $(\mathrm{C} 1)$ is automatic.)

\begin{tabular}{|c|c|c|c|}
\hline$M_{n}$ & convexity (C1) & connectedness of nonzero level sets (C2) & openness (C3) \\
\hline$n=1$ & $\checkmark$ & $\checkmark$ & $\checkmark$ \\
$n \geq 2$ & $\checkmark$ & $\times$ & $\checkmark$ \\
\hline
\end{tabular}

Table 3

\section{References}

[1] C Albert, Le théorème de réduction de Marsden-Weinstein en géométrie cosymplectique et de contact, J. Geom. Phys. 6 (1989) 627-649 MR1076705

[2] MF Atiyah, Convexity and commuting Hamiltonians, Bull. London Math. Soc. 14 (1982) 1-15 MR642416

[3] A Banyaga, P Molino, Géométrie des formes de contact complètement intégrables de type toriques, from: "Séminaire Gaston Darboux de Géométrie et Topologie Différentielle, 1991-1992 (Montpellier)", Univ. Montpellier II (1993) 1-25 MR1223155 


\begin{tabular}{|ccc|c|c|c|}
\hline$M_{[a, b]}, b=b^{\prime}+2 \pi k$ & & $(\mathrm{C} 1)$ & $(\mathrm{C} 2)$ & $(\mathrm{C} 3)$ \\
\hline$a=-\pi / 2$, & $-\pi / 2<b^{\prime} \leq \pi / 2$, & $k=0$ & $\checkmark$ & $\checkmark$ & $\checkmark$ \\
& & $k \geq 1$ & $\checkmark$ & $\times$ & $\times$ \\
\hline$a=-\pi / 2$, & $\pi / 2<b^{\prime} \leq 3 \pi / 2$, & $k=0$ & $\checkmark$ & $\checkmark$ & $\times$ \\
& & $k \geq 1$ & $\checkmark$ & $\times$ & $\times$ \\
\hline$-\pi / 2<a<\pi / 2$, & $a<b^{\prime}<\pi / 2$, & $k=0$ & $\checkmark$ & $\checkmark$ & $\checkmark$ \\
& & $k \geq 1$ & $\checkmark$ & $\times$ & $\checkmark$ \\
\hline$-\pi / 2<a<\pi / 2$, & $b^{\prime}=\pi / 2$, & $k=0$ & $\checkmark$ & $\checkmark$ & $\checkmark$ \\
& & $k \geq 1$ & $\checkmark$ & $\times$ & $\times$ \\
\hline$-\pi / 2<a<\pi / 2$, & $\pi / 2<b^{\prime}<3 \pi / 2$, & $k=0$ & $\checkmark$ & $\checkmark$ & $\checkmark$ \\
& & $k \geq 1$ & $\checkmark$ & $\times$ & $\checkmark$ \\
\hline$-\pi / 2<a<\pi / 2$, & $b^{\prime}=3 \pi / 2$, & $k=0$ & $\checkmark$ & $\checkmark$ & $\times$ \\
& & $k \geq 1$ & $\checkmark$ & $\times$ & $\times$ \\
\hline$-\pi / 2<a<\pi / 2$, & $3 \pi / 2<b^{\prime} \leq a+2 \pi$, & $k \geq 0$ & $\checkmark$ & $\times$ & $\checkmark$ \\
\hline
\end{tabular}

Table 4

[4] A Barvinok, A course in convexity, Graduate Studies in Math. 54, Amer. Math. Soc. (2002) MR1940576

[5] M Berger, Geometry. I, Universitext, Springer, Berlin (1987) MR882541 Translated from the French by M Cole and S Levy

[6] M Berger, Geometry. II, Universitext, Springer, Berlin (1987) MR882916 Translated from the French by M Cole and S Levy

[7] P Birtea, J-P Ortega, T S Ratiu, A local-to-global principle for convexity in metric spaces, J. Lie Theory 18 (2008) 445-469 MR2431127

[8] P Birtea, J-P Ortega, T S Ratiu, Openness and convexity for momentum maps, Trans. Amer. Math. Soc. 361 (2009) 603-630 MR2452817

[9] C Bjorndahl, Y Karshon, Revisiting Tietze-Nakajima - local and global convexity for maps, to appear in the Canad. J. Math. arXiv:math/0701745v4

[10] C P Boyer, K Galicki, A note on toric contact geometry, J. Geom. Phys. 35 (2000) 288-298 MR1780757

[11] M R Bridson, A Haefliger, Metric spaces of non-positive curvature, Grund. der Math. Wissenschaften 319, Springer, Berlin (1999) MR1744486

[12] M Condevaux, P Dazord, P Molino, Géométrie du moment, from: “Travaux du Séminaire Sud-Rhodanien de Géométrie, I”, Publ. Dép. Math. Nouvelle Sér. B 88, Univ. Claude-Bernard, Lyon (1988) 131-160 MR1040871 
[13] L Danzer, B Grünbaum, V Klee, Helly's theorem and its relatives, from: "Proc. Sympos. Pure Math., Vol. VII”, Amer. Math. Soc. (1963) 101-180 MR0157289

[14] H Geiges, Constructions of contact manifolds, Math. Proc. Cambridge Philos. Soc. 121 (1997) 455-464 MR1434654

[15] H Geiges, An introduction to contact topology, Cambridge Studies in Advanced Math. 109, Cambridge Univ. Press (2008) MR2397738

[16] V Guillemin, R Sjamaar, Convexity properties of Hamiltonian group actions, CRM Monogr. Ser. 26, Amer. Math. Soc. (2005) MR2175783

[17] V Guillemin, S Sternberg, Convexity properties of the moment mapping, Invent. Math. 67 (1982) 491-513 MR664117

[18] V Guillemin, S Sternberg, Homogeneous quantization and multiplicities of group representations, J. Funct. Anal. 47 (1982) 344-380 MR665022

[19] J Hilgert, K-H Neeb, W Plank, Symplectic convexity theorems, Sem. Sophus Lie 3 (1993) 123-135 MR1270171

[20] J Hilgert, K-H Neeb, W Plank, Symplectic convexity theorems and coadjoint orbits, Compositio Math. 94 (1994) 129-180 MR1302314

[21] Y Kamishima, T Tsuboi, CR-structures on Seifert manifolds, Invent. Math. 104 (1991) 149-163 MR1094049

[22] F Knop, Convexity of Hamiltonian manifolds, J. Lie Theory 12 (2002) 571-582 MR1923787

[23] E Lerman, Contact cuts, Israel J. Math. 124 (2001) 77-92 MR1856505

[24] E Lerman, A convexity theorem for torus actions on contact manifolds, Illinois J. Math. 46 (2002) 171-184 MR1936083

[25] E Lerman, Contact toric manifolds, J. Symplectic Geom. 1 (2003) 785-828 MR2039164

[26] E Lerman, E Meinrenken, S Tolman, C Woodward, Nonabelian convexity by symplectic cuts, Topology 37 (1998) 245-259 MR1489203

[27] E Lerman, C Willett, The topological structure of contact and symplectic quotients, Internat. Math. Res. Notices (2001) 33-52 MR1809496

[28] F Loose, Reduction in contact geometry, J. Lie Theory 11 (2001) 9-22 MR1828281

[29] S F B de Moraes, C Tomei, Moment maps on symplectic cones, Pacific J. Math. 181 (1997) 357-375 MR1486536

[30] S Nakajima, Über konvexe Kurven und Flächen, Tohoku Math. J. 29 (1928) 227-230

[31] H Nozawa, Five dimensional $K$-contact manifolds of rank 2 arXiv:0907.0208v1

[32] E Prato, Convexity properties of the moment map for certain non-compact manifolds, Comm. Anal. Geom. 2 (1994) 267-278 MR1312689 
[33] C V Robinson, Spherical theorems of Helly type and congruence indices of spherical caps, Amer. J. Math. 64 (1942) 260-272 MR0006420

[34] R Sjamaar, Convexity properties of the moment mapping re-examined, Adv. Math. 138 (1998) 46-91 MR1645052

[35] H Tietze, Über Konvexheit im kleinen und im großen und über gewisse den Punkten einer Menge zugeordnete Dimensionszahlen, Math. Z. 28 (1928) 697-707 MR1544985

[36] C Willett, Contact reduction, Trans. Amer. Math. Soc. 354 (2002) 4245-4260 MR1926873

Department of Mathematics, National Cheng Kung University

Tainan 701, Taiwan

Department of Mathematics, University of Toronto

Toronto, Ontario M5S 2E4, Canada

riverch@mail.ncku.edu.tw, karshon@math.toronto.edu

http://www . math.toronto.edu/karshon/

Received: 5 November 2009 Revised: 25 February 2010 